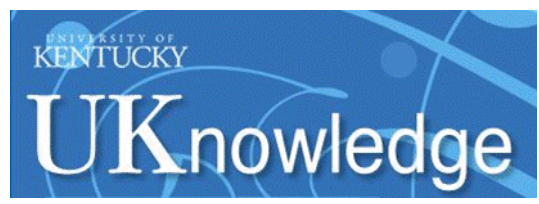

University of Kentucky

UKnowledge

\title{
The Dynamics of Stellar Outflows Dominated by Interaction of Dust and Radiation
}

\author{
Nathan Netzer \\ University of Kentucky \\ Moshe Elitzur \\ University of Kentucky, moshe@pa.uky.edu
}

Follow this and additional works at: https://uknowledge.uky.edu/physastron_facpub

Part of the Astrophysics and Astronomy Commons, and the Physics Commons

Right click to open a feedback form in a new tab to let us know how this document benefits you.

\section{Repository Citation}

Netzer, Nathan and Elitzur, Moshe, "The Dynamics of Stellar Outflows Dominated by Interaction of Dust and Radiation" (1993). Physics and Astronomy Faculty Publications. 227.

https://uknowledge.uky.edu/physastron_facpub/227

This Article is brought to you for free and open access by the Physics and Astronomy at UKnowledge. It has been accepted for inclusion in Physics and Astronomy Faculty Publications by an authorized administrator of UKnowledge. For more information, please contact UKnowledge@lsv.uky.edu. 


\section{The Dynamics of Stellar Outflows Dominated by Interaction of Dust and Radiation Digital Object Identifier (DOI) http://dx.doi.org/10.1086/172787 \\ Notes/Citation Information \\ Published in The Astrophysical Journal, v. 410, no. 2, p. 701-713. \\ (c) 1993. The American Astronomical Society. All rights reserved. \\ The copyright holder has granted permission for posting the article here.}




\title{
THE DYNAMICS OF STELLAR OUTFLOWS DOMINATED BY INTERACTION OF DUST AND RADIATION
}

\author{
NATHAN NeTZER ${ }^{1}$ AND MOSHE ElitzUr \\ Department of Physics and Astronomy, University of Kentucky, Lexington, KY 40506-0055 \\ Received 1992 January 20; accepted 1992 December 17
}

\begin{abstract}
Wind outflow around a late-type star driven by radiation pressure on dust grains is investigated in detail. The equation of motion for the outflow coupled with the equation of radiative transfer is solved treating the circumstellar envelope, which consists of gas and dust, as a two-component fluid. Because of the drift of the dust particles through the gas, the dust-to-gas ratio varies with distance even if grain formation is a prompt process. The coupling between dust and gas weakens as the mass-loss rate decreases until finally the rate of momentum transfer to the gas is insufficient to overcome gravity when the mass-loss rate is too low. This results in a lower limit, on the order of $10^{-7} M_{\odot} \mathrm{yr}^{-1}$, to the possible mass-loss rates that can be driven by radiation pressure on dust. Radiative transfer considerations pose a bound on the capability of the radiation field to overcome gravity also in the opposite limit of large mass-loss rates. Due to the difference in optical properties between carbon stars and oxygen stars, these considerations produce a meaningful upper limit on the mass-loss rate, of several times $10^{-4} M_{\odot} \mathrm{yr}^{-1}$, only for carbon stars.

Subject headings: circumstellar matter - dust, extinction - ISM : jets and outflows - stars: mass loss
\end{abstract}

\section{INTRODUCTION}

Radiation pressure on dust grains is widely believed to be the driving force of stellar outflows from late-type giants and supergiants (cf. Salpeter 1974; Goldreich \& Scoville 1976). As the gas cools below a certain temperature (around $1000 \mathrm{~K}$ ), heavy elements condense. Radiation pressure acts on dust grains, and momentum is transferred to the gas molecules by collisions. A complete solution of the dynamics of the outflows must involve a solution of the radiative transfer problem in spherical dust clouds, a problem that was treated in a number of works in the past (Leung 1975; Rowan-Robinson 1980; Rowan-Robinson \& Harris 1982, 1983; Adams \& Shu 1986). All of these studies assumed a given mass density distribution, which in the case of circumstellar envelopes implies a given velocity field. The latter, however, is determined by the radiation pressure exerted on the envelope by the radiation originating in the central star. Thus the radiative transfer problem and the dynamical problem are inherently coupled.

The importance of radiative transfer effects in stellar outflows is manifested by the evidence for a gradual acceleration in certain red supergiants (Chapman \& Cohen 1986; Bowers \& Johnston 1990). A possible dependence of the dust opacity on position can arise not only from grain growth, but also because the envelope is a two fluid system. It consists of dust and gas, and the dust moves supersonically within the gas, reaching its terminal velocity much faster than does the gas. Thus the dustto-gas ratio is reduced relative to its rest value by the ratio of the two outflow velocities, which is not constant throughout the envelope. Although this relative motion has been mentioned in several works (cf. Gilman 1972; Kwok 1975), its impact on the opacity has not been incorporated. In this work, we attempt to address this problem within the full solution of the dynamics and radiative transfer coupled together.

The momentum transfer problem is presented in $\S 2$. In $\S 3$ the equations of radiative transfer in the moment form are solved by separation of the stellar radiation field from the

\footnotetext{
1 Postal address: ORT-Braude College, P.O. Box 78, Karmiel 20101, Israel.
}

diffuse infrared field of the envelope. In $\S 4$ the dust formation and properties are discussed, as well as the dust drift. In $\S 5$ the results of the calculations for carbon and oxygen stars are presented. In $\S 6$ we discuss a possible explanation for outflow velocity gradients in supergiants, and in $\S 7$ we summarize the general conclusions.

\section{THE MOMENTUM TRANSFER PROBLEM}

The equation of motion of the circumstellar envelope is given by

$$
v \frac{d v}{d r}=-\frac{1}{\rho} \frac{d P}{d r}+\frac{1}{c} \int_{0}^{\infty} \chi_{v} F_{v} d v-\frac{G M_{*}}{r^{2}}
$$

where $v, \rho$, and $P$ are, respectively, the gas velocity, density, and pressure; $\chi_{v}$ is the opacity per unit mass at frequency $v ; F_{v}$ is the radiative radial flux; and $M_{*}$ is the stellar mass. The second term on the right-hand side expresses the radiation pressure. This is the dominant force in the envelope and the only term that will be retained in this section. The first term, the gas pressure gradient, is negligible in general: if we replace $P$ by $\rho v_{s}^{2}$, where $v_{s}$ is the isothermal sound velocity, then with the aid of mass conservation $\rho=\dot{M} /\left(4 \pi r^{2} v\right)$,

$$
\frac{1}{\rho} \frac{d}{d r}\left(\rho v_{s}^{2}\right)=2 v_{s} \frac{d v_{s}}{d r}-\frac{v_{s}^{2}}{v} \frac{d v}{d r}-\frac{2 v_{s}^{2}}{r} .
$$

All the terms on the right-hand side are much smaller than $v d v / d r$ for the highly supersonic outflows discussed here. The gravitational deceleration, too, can be ignored in most of the important cases and is omitted in this section to simplify the discussion.

Define the flux mean of the opacity

$$
\chi_{F}=\frac{1}{F} \int \chi_{v} F_{v} d v
$$

where $F=\int F_{v} d v$ is the overall radiative flux. Then, utilizing 
flux conservation

$$
F=\frac{L_{*}}{4 \pi r^{2}},
$$

where $L_{*}$ is the stellar bolometric luminosity, we have

$$
\int \chi_{v} F_{v} d v=\chi_{F} \frac{L_{*}}{4 \pi r^{2}}
$$

and hence

$$
v \frac{d v}{d r}=\chi_{F} \frac{L_{*}}{4 \pi r^{2} c} .
$$

The mean opacity $\chi_{F}$ can vary with position due to possible changes in grain size and spatial density relative to the density of gas, and due to variations in the radiation spectral distribution (resulting from radiation absorption and reemission), which occur if the envelope is optically thick. Even when these effects can be neglected, $\chi_{F}$ still can vary due to the two-fluid character of the problem, which will be discussed below.

Define $d \tau_{F}=\chi_{F} \rho d r$, so $\tau_{F}$ is the flux-mean optical depth from the inner edge of the envelope outward. Then equation (6) becomes

$$
\dot{M} \frac{d v}{d \tau_{F}}=\frac{L_{*}}{c},
$$

and integrating we get

$$
\dot{M}\left[v(r)-v\left(r_{0}\right)\right]=\frac{L_{*}}{c} \tau_{F}(r) .
$$

This result shows that at every point in the envelope, the velocity is linearly related to the flux-mean optical depth $\tau_{F}$. In deriving this relation, we used flux conservation, equation (4), which is not strictly obeyed since the total flux decreases throughout the envelope due to conversion of radiative energy into kinetic energy. Flux conservation, however, is an excellent approximation in the cases discussed here. Strict conservation implies that $L_{*}$ should be replaced everywhere with $L_{*}$ $-\frac{1}{2} \dot{M}\left(v^{2}-v_{0}^{2}\right)$, and equation (8) would then contain a term $\dot{M}\left(v^{2}-v_{0}^{2}\right) \tau_{F} / 2 c$ on the right-hand side. The relative correction is of order $\tau_{F} v / c$, typically much less than 1 ; only a minute fraction of the stellar luminosity is converted into kinetic energy.

Assuming that sufficiently far from the star the velocity increases greatly above its initial value, we can ignore $v_{0}$, and equation (8) becomes

$$
\dot{M} v(r)=\frac{L_{*}}{c} \tau_{F}(r),
$$

or, numerically,

$$
\dot{M}=2 \times 10^{-5} \tau_{F} \frac{L_{4}}{v_{10}} M_{\odot} \mathrm{yr}^{-1} .
$$

Here $L_{4}$ is the luminosity in units of $10^{4}$ solar luminosities and $v_{10}$ is the velocity in units of $10 \mathrm{~km} \mathrm{~s}^{-1}$. Equation (9) expresses a well-known relation (cf. Knapp 1986). It has often been referred to as a qualitative result, applicable only when the optical depth is small, in which case it is the probability for a photon to interact on its way out. Thus $L_{*} / c$ has been assumed to be the maximum possible value for momentum transfer rate, providing an upper limit on the possible mass-loss rate. This is not the case, however. As long as the various assumptions listed above are met, equation (9) is an exact relation, not merely a qualitative approximation, provided that the optical depth is taken as the flux-mean optical depth; this has also been recognized by Gail \& Sedlmayr (1987). Moreover, it is a general result, not merely restricted to small optical depths, and thus $L_{*} / c$ is not an upper limit on the rate of momentum transfer from the stellar radiation to the envelope. This does not violate momentum conservation, as might appear at first. The reason is that the common consideration about momentum conservation assumes, implicitly, that a photon interacts with a dust grain only once. But when the envelope is optically thick this is no longer the case. A photon interacts several times on its way out and this multiple scattering effect is contained in $\tau_{F}$. On the average, the envelope stores $\tau_{F}$ photons for each photon emitted by the star, since $\tau_{F}$ is the mean number of scatterings a photon undergoes on its way out, so the stored momentum is multiplied accordingly. This is best illustrated by equation (8): $\dot{M} v(r)$ is simply the rate at which mechanical momentum is transferred outward across a spherical surface with radius $r$. Therefore equation (8) shows that the overall amount of momentum stored in a thick shell bounded by the radii $r_{0}$ and $r$ increases in linear proportion to the shell's mean optical depth, $\tau_{F}(r)$.

From this discussion it follows that $L_{*} /\left(v_{e} c\right)$, where $v_{e}$ is the envelope's final expansion velocity, is not an upper limit, only a rough estimate for the order of magnitude of the mass-loss rate. This invalidates a commonly used argument against radiation pressure as a possible mechanism for acceleration of protostellar jets (e.g., Welch 1989). In addition, the large scatter observed for carbon stars in the correlation between $L_{*} /\left(v_{e} c\right)$ and $\dot{M}$ (Knapp et al. 1982) is, therefore, not a valid argument against radiation pressure on dust grains as the mechanism for mass-loss.

Utilizing mass conservation, the flux-mean optical depth is

$$
\tau_{F}(r)=\frac{\dot{M}}{4 \pi} \int_{r_{0}}^{r} \frac{\chi_{F}(r)}{r^{2} v(r)} d r .
$$

Therefore, the explicit dependence on $\dot{M}$ disappears from equation (9) since both sides of the equation are proportional to it. The only possible dependence on $\dot{M}$ is indirect in that it can affect $\chi_{F}$ through the averaging procedure (eq. [3]) because of its impact on the radiation spectral shape through the overall solution. To lowest order, radiation pressure does not provide any constraint on the mass-loss rate $\dot{M}$; all the material fed in at the dust formation point, by whatever process is lifting it to the radius $r_{0}$, will be driven away by radiation pressure. The only constraint the operation of this mechanism provides is on the velocity profile. This is easily seen by inserting equation (11) back into equation (8) leading to

$$
v(r)=v\left(r_{0}\right)+\frac{L_{*}}{4 \pi c} \int_{r_{0}}^{r} \frac{\chi_{F}(r)}{r^{2} v(r)} d r .
$$

The above discussion breaks down in the limit in which the fraction of radiative energy converted into kinetic energy is not negligible. As $\dot{M}$ is increased, more radiative energy is converted into kinetic energy and eventually the flux conservation assumption (eq. [4]), on which the above derivation is based, no longer applies. Typically, this will occur when $\tau_{F} v / c$ begins to approach unity. Late-type stars are always far below this limit.

It should be noted that even in the case of prompt dust 
formation and negligible grain growth afterward, $\chi_{F}$ still does vary with position in general. This quantity involves an average weighted with the spectral distribution $F_{v} / F$ (eq. [3]), and this weight function varies with radius as radiation is absorbed and reemitted, shifting toward the red. Thus the dynamical problem and radiative transfer are inherently coupled. The solution of this combined problem is discussed in the next section.

\section{THE CENTRAL STAR AND DIFFUSE FIELD EQUATIONS}

\subsection{Moment Equations}

Along with the equation of motion, equation (1), the first moment of the equation of radiative transfer (cf. Schwarzschild 1958) provides the flux relation

$$
\frac{1}{r^{2}} \frac{d}{d r}\left[r^{2} F_{v}(r)\right]=\chi_{v}^{a} \rho\left[4 \pi B_{v}(T)-c u_{v}\right],
$$

where $u_{v}$ is the energy spectral density and $\chi_{v}^{a}$ is the opacity per unit mass due to absorption; that is, the total opacity per unit mass is written as

$$
\chi_{v}=\chi_{v}^{a}+\chi_{v}^{s}
$$

and $\chi_{v}^{s}$ is the scattering opacity. The second moment provides the radiation pressure equation

$$
\frac{d P_{v}}{d r}+\frac{3 P_{v}-u_{v}}{r}+\frac{\chi_{v} \rho}{c} F_{v}=0,
$$

when $P_{v}$ is the radial-radial term of the stress tensor, expressing the radiation pressure when the radiation field is isotropic. It should be noticed that equation (13) describes the change in the spectral distribution, which is affected by absorption but not by scattering and hence $\chi_{v}^{a}$ appears there. The third term on the left-hand side of equation (15), on the other hand, is a force term and hence scattering contributes too. We assume isotropic scattering.

As is well known, the above set of equations cannot be closed. Higher moment equations can be easily generated, but they simply lead to more relations in which the number of unknown variables exceeds the number of equations. This problem can be avoided only if an additional relationship among the first three moments is identified. Such a relationship is easily recognized in two limiting cases: (1) When the radiation field is isotropic (the optically thick case), $P_{v}=u_{v} / 3$. The second term in equation (15) then vanishes and the density of radial force exerted by the radiation field is the pressure gradient. (2) When the radiation field is radial, as in the case of a point source, we can use the approximation $F=c P_{r}=c u=$ $L / 4 \pi r^{2}$, where $P_{r}=\int P_{v} d v$ is the overall radiation pressure. Assuming that $L$ is the stellar radiation that has not been extinguished (and hence is variable) we get from equation (13)

$$
\frac{d L}{d r}+\rho \chi_{F} L=0 \Rightarrow L=L_{*} \exp \left(-\int \chi_{F} \rho d r\right),
$$

which expresses the exponential extinction, as well as the fact that the photons lost from the radial beam cause the driving force.

Since the diffuse radiation from the dust is neither isotropic nor a point source, the general case of a circumstellar envelope does not correspond to either of the above two limits and another relation among the first three moments is required. For that we shall separate the radiation field into two com- ponents: The first component corresponds to photons emanating from the central star that have been neither absorbed nor scattered by dust. Since the star is assumed to be a point source, this component is radial and satisfies

$$
P_{v}^{S}=u_{v}^{S}=\frac{F_{v}^{S}}{c}=\frac{\pi R_{*}^{2}}{c r^{2}} B_{v}\left(T_{e}\right) \exp \left(-\tau_{v}\right),
$$

where $\tau_{v}$ is the optical depth measured from the inner edge of the envelope along the radius. Once the density profile is known from the equation of motion, $\tau_{v}$ can be readily calculated. The second component of the radiation field is the diffuse radiation, consisting of photons emitted or scattered by dust grains. The pressure, flux, and energy density of the diffuse field will be denoted by $P_{v}^{d}, F_{v}^{d}$, and $u_{v}^{d}$, respectively. From equation (13) we obtain

$$
\frac{1}{r^{2}} \frac{d}{d r}\left[r^{2}\left(F_{v}^{S}+F_{v}^{d}\right)\right]=\chi_{v}^{a} \rho\left[4 \pi B_{v}(T)-c u_{v}^{S}-c u_{v}^{d}\right] .
$$

Then with the aid of equations (14) and (17) we get

$$
\begin{aligned}
\frac{1}{r^{2}} \frac{d}{d r}\left(r^{2} F_{v}^{d}\right)= & \chi_{v}^{a} \rho\left[4 \pi B_{v}(T)-c u_{v}^{d}\right] \\
& +\chi_{v}^{s} \rho \frac{\pi R_{*}^{2}}{r^{2}} B_{v}\left(T_{e}\right) \exp \left(-\tau_{v}\right) .
\end{aligned}
$$

This is the source equation for the diffuse radiation, which shows that it has two sources (thermal emission of grains and scattering of photons from the central star) and a sink (absorption by dust grains). Similarly, from equation (15) we get

$$
\frac{d P_{v}^{d}}{d r}+\frac{3 P_{v}^{d}-u_{v}^{d}}{r}+\frac{\chi_{v} \rho}{c} F_{v}^{d}=0
$$

These results show that the forces exerted by each component of the radiation field can be treated independent of the other.

\subsection{The Closure Relation}

As mentioned above, an additional relation among the energy density, the radiative flux, and the radiation pressure is needed to close the system of equations, a problem involving only the diffuse field since the stellar component is known. As a relation valid everywhere, Adams \& Shu (1986) introduced the equation

$$
u=3 P_{r}-\frac{q}{c} F
$$

where $q$ is a continuous function, ranging from 2 for optically thin regions $\left(u=P_{r}=F / c\right)$ to 0 for optically thick ones $(u=$ $\left.3 P_{r}\right)$; the exact value of $q$ is determined from overall flux conservation at every point. The Adams \& Shu relation involves the frequency-integrated energy density, flux, and pressure. We extend this relation to spectral densities

$$
u_{v}^{d}=3 P_{v}^{d}-\frac{q_{v}}{c} F_{v}^{d},
$$

and parameterize $q_{v}$ according to

$$
q_{v}=2\left[1-\exp \left(-\alpha \tau_{v}\right)\right] .
$$

Here $\alpha$ is a dimensionless parameter (typically $\alpha \gtrsim 1$ ) determined from a best fit to flux conservation at every point. 
Note that $\tau_{v}$ is measured from the shell's inner edge outward. When the wind's overall optical depth is large, small values of $\tau_{v}$ correspond to the optically thick (inner) parts of the wind while large $\tau_{v}$ mark the optically thin (outer) parts. It turns out that $q_{v}=2$ throughout the entire envelope and for all frequencies is a reasonable approximation in many cases. The reason is that in the optically thick region, where the diffuse radiation is isotropic, $F_{v}^{d} / c \ll u_{v}^{d}$, and hence the results are not very sensitive to the exact value of $q_{v}$.

This approach enables us to complete the system of equations describing the full problem. Substituting equation (21) into equations (17) and (18) we get

$$
\begin{aligned}
\frac{1}{r^{2}} \frac{d}{d r}\left(r^{2} F_{v}^{d}\right)= & \chi_{v}^{a} \rho\left[4 \pi B_{v}(T)-3 c P_{v}^{d}+q_{v} F_{v}^{d}\right] \\
& +\chi_{v}^{s} \rho \frac{\pi R_{*}^{2}}{r^{2}} B_{v}\left(T_{e}\right) \exp \left(-\tau_{v}\right) \\
c \frac{d P_{v}^{d}}{d r} & =-\left(\frac{q_{v}}{r}+\chi_{v} \rho\right) F_{v}^{d},
\end{aligned}
$$

along with the radiative relations

$$
\begin{gathered}
F_{v}^{S}=c u_{v}^{S}=\frac{\pi R_{*}^{2}}{r^{2}} B_{v}\left(T_{e}\right) \exp \left(-\tau_{v}\right) \\
\frac{d \tau_{v}^{a}}{d r}=\chi_{v}^{a} \rho, \\
\frac{d \tau_{v}^{s}}{d r}=\chi_{v}^{s} \rho,
\end{gathered}
$$

and the equation of motion

$$
v \frac{d v}{d r}=\frac{1}{c} \int \chi_{v}\left[F_{v}^{d}+\frac{\pi R_{0}^{2}}{r^{2}} B_{v}\left(T_{e}\right) \exp \left(-\tau_{v}\right)\right] d v-\frac{G M_{*}}{r^{2}} .
$$

The only quantity still undetermined is the dust temperature $T$. The required relation for it can be obtained from integration over frequency of the flux conservation relation, equation (13), leading to

$$
\chi_{P} a T^{4}=\chi_{u} u
$$

Here $\chi_{P}$ and $\chi_{u}$ are mean absorption opacities obtained by averaging $\chi_{v}^{a}$ over frequencies (as in eq. [3]) with the Planck function and the energy spectral density, respectively. This relation can be used to determine the temperature.

\subsection{The Solution Scheme; Boundary Conditions}

The system of equations is now fully defined. In order to solve it we divide the shell into radial zones, and at each radius we build a grid of $n$ frequencies. Equations (23)-(28) then provide a set of $4 n+2$ differential equations in $4 n+2$ unknown functions, namely, $2 n$ optical depths for scattering and absorption, $n$ differential pressures, $n$ differential fluxes, the outflow velocity, and the temperature. In the numerical solutions we typically use $n=80$ frequency points. The radial integration is carried out using a predictor-corrector method and the integration step is defined at each point so that the convergence is fast and a minimum of time is required for the entire process.

The boundary conditions are specified on $R_{0}$, the inner envelope radius, as follows:
All the optical depths $\tau_{v}^{a}$ and $\tau_{v}^{s}$, which are measured from inside outward, are zero at $R_{0}$. The initial value of the outflow velocity is given some arbitrary small magnitude since its actual value is determined by processes in the stellar atmosphere that are outside the scope of this work. Fortunately, the results are almost independent of the exact value of this initial guess.

The inner radius $R_{0}$ is determined from the assumption that this is the dust formation point. The temperature at this radius then corresponds to the dust formation temperature and can be determined from the radiative equilibrium relation, equation (28). Most of the radiation in the inner hollow cavity corresponds to the stellar contribution, but at high mass-loss rates there is also a significant diffuse infrared component that originates from the dusty envelope. From equation (28),

$$
4 \pi \int_{0}^{\infty} \chi_{v}^{a} B_{v}\left(T_{0}\right) d v=\int_{0}^{\infty} \chi_{v}^{a} \frac{R_{*}^{2}}{4 R_{0}^{2}} B_{v}\left(T_{e}\right) d v+c \int_{0}^{\infty} \chi_{v}^{a} u_{v}^{d}\left(R_{0}\right) d v,
$$

where $T_{0}$ is the dust formation temperature, taken as $800-900$ $K$. This equation involves the remaining boundary conditions that need to be specified, namely, the flux, $F_{v}^{d}$, and pressure, $P_{v}^{d}$, of the diffuse radiation field in the cavity. We can reasonably assume this radiation field to be constant. The reason is that when the dust shell is optically thick, the radiation field in the central cavity is similar to that of a blackbody. And when the envelope is optically thin, most of the radiation is contributed by remote shells, whose size is much larger then the size of the cavity. Therefore, in the first iteration for the diffuse radiation within the cavity we assume

$$
c P_{v}^{d}=\frac{4 \pi}{3} B_{v}\left(T_{i}\right)
$$

where $T_{i}$ is an arbitrary initial guess for the cavity's temperature, constrained only by the requirement that it is lower than the assumed dust formation temperature. This completes the specification of all the necessary boundary conditions and enables the finding of a solution, from which we calculate the cavity's diffuse energy density for the next iteration

$$
c u_{v}^{d}=\int_{R_{0}}^{R_{\infty}} \chi_{v}^{a} \frac{\dot{M}}{4 \pi r^{2} v} B_{v}(T) \exp \left(-\tau_{v}^{a}-\tau_{v}^{s}\right) d r
$$

where $R_{\infty}$ is a large cutoff radius. This result provides the energy density at the center, assumed valid throughout the entire cavity, which is used for the next iteration, and so forth. Evidently, the dust formation radius does vary somewhat from one iteration to the next, but convergence is usually very fast. For both silicate and graphite grains this radius turns out to be $\sim 4-6$ stellar radii if the photospheric temperature is assumed in the range between 2000 and $3000 \mathrm{~K}$.

As a test of our solution procedure, we have solved the wind structure using frequency-independent (gray) dust opacity as an input. This unrealistic case admits a simple analytic solution (see eq. [38] below), and we have verified that our numerical scheme properly reproduces this solution.

\section{DUST PROPERTIES}

\subsection{Optical Properties of the Dust}

The dust opacity is an essential ingredient that enters the equations as described in the previous chapter. The dust optical properties depend on its chemical composition. When 
the dust consists of silicates, as is the case in outflows of oxygen stars, the optical properties are dominated by the absorption peaks at $\lambda=9.7 \mu \mathrm{m}$ and $\lambda=18 \mu \mathrm{m}$. Outflows in carbon stars, on the other hand, are characterized by an absorption opacity that decreases monotonically as a function of wavelength over most of the infrared range. The relative abundance of heavy elements determines the amount of mass in grains, and the grain sizes determine their number. If we assume for simplicity that the dust grains are spherical, the opacity is given by

$$
\chi(\lambda)=\frac{1}{\rho} \int Q_{\lambda}(a) \pi a^{2} n(a) d a,
$$

where $n(a)$ is the number density of dust particles per unit grain radius $a$. When $a \ll \lambda$, the absorption efficiency is given by the dipole approximation (van de Hulst 1957)

$$
\begin{aligned}
& Q_{\lambda}^{a}(a)=-4 \frac{2 \pi a}{\lambda} \operatorname{Im}\left(\frac{\epsilon-1}{\epsilon+2}\right), \\
& Q_{\lambda}^{s}(a)=\frac{8}{3}\left(\frac{2 \pi a}{\lambda}\right)^{4}\left|\frac{\epsilon-1}{\epsilon+2}\right|^{2} .
\end{aligned}
$$

Here $\epsilon$ is the dielectric function of the grain material. Although it usually depends only on wavelength, for graphite grains it may depend also on grain size (Draine \& Lee 1984). When $a \gg \lambda$ the total extinction efficiency is 2 , and we shall assume that both the absorption and scattering coefficients separately become constant in this regime. then

If the fraction of mass incorporated in dust grains is fixed

$$
\int a^{3} n(a) d a=\text { const } \times \rho,
$$

where the proportionality constant is related to the cosmic dust to gas ratio. From equations (32)-(34) it then follows that, when the dust consists mostly of small grains, the absorption opacity becomes independent of the functional form of the size distribution $n(a)$ and scattering becomes negligible. On the other hand, when the grains are large, both opacities decrease as the grain size increases. The dynamics of the outflow modifies this situation, as discussed below.

\subsection{Dust Drift}

Another important issue is the variation of the opacity with radius at each frequency. It is usually assumed that dust formation is an abrupt process that occurs as a phase transition when the temperature decreases below a certain value. This would imply that, beyond this point and at each frequency, the opacity per unit mass becomes independent of radius. Indeed, this assumption is frequently made (e.g., Goldreich \& Scoville 1976). There are, however, considerable uncertainties. At small radii, collisions among grains can lead to coalescence or breakup. In addition, not all the species condense at the same temperature and grains can keep growing after their initial formation. Even though in a steady outflow one might expect grain growth to stop at large radii because of kinetic considerations (the drop in gas density), it is quite likely that clumps are present and hence continued accretion is feasible. Unfortunately, no adequate modeling has yet been performed to describe clumps and their effect in a quantitative manner.

All of these uncertainties are dwarfed by another effect. The dust flows out faster than the gas, resulting in a twocomponent fluid. Therefore the dust-to-gas ratio, and hence the opacity too, is reduced by a factor $v / v_{d}$, where $v$ and $v_{d}$ are correspondingly the gas and dust outflow velocities (note that $v$ is the observed envelope outflow velocity). To account for these different velocities, the gas and dust must be treated separately in the dynamical equations.

Three forces act on a dust grain in a circumstellar envelope: the radiation pressure, the gravitational pull of the star, and the drag force of the gas. The equation of motion of a single dust grain is thus

$$
v_{d} \frac{d v_{d}}{d r}=\frac{Q_{F} L_{*} \pi a^{2}}{4 \pi r^{2} m_{d} c}-\frac{G M_{*}}{r^{2}}-\frac{\dot{M}}{4 \pi r^{2} m_{d} v}\left(v_{d}-v\right)^{2} \pi a^{2},
$$

where $m_{d}$ is the grain mass and $Q_{F}$ is the flux mean of the extinction efficiency (the dust drift relative to the gas is assumed to be highly supersonic, which is a self-consistent assumption). Correspondingly, the equation for the gas is

$$
v \frac{d v}{d r}=-\frac{G M_{*}}{r^{2}}+\frac{\dot{M} n_{d}}{4 \pi r^{2} v_{d} \rho}\left(v_{d}-v\right)^{2} \pi a^{2},
$$

where $n_{d}$ is the volume density of dust grains. This equation contains a variation of the dust-to-gas ratio in proportion to $v / v_{d}$, on account of the different velocities for dust and gas. From these equations it is easy to show that the drift velocity, $v_{\text {drift }}=v_{d}-v$, reaches its terminal value much faster than the gas outflow velocity (see Gilman 1972). This steady state drift velocity is given by

$$
v_{\mathrm{drift}}=\left(\frac{Q_{F} L_{*} v}{\dot{M} c}\right)^{1 / 2}
$$

Therefore, in regions where $v$ is still small but $v_{\text {drift }}$ is already close to its terminal value, the factor $v / v_{d}$ leads to a considerable decrease in opacity. This is the case in the innermost parts of the envelope, where otherwise the above-mentioned uncertainties regarding the opacity are the most severe. This effect is also stronest in thin envelopes.

Since $Q_{F}$ in equation (37) depends on $a$, grains of different sizes drift at different velocities and any assumption about an initial grain size distribution does not hold throughout the entire envelope. For simplicity we shall only consider a single size for all grains.

\section{THE SOLUTION}

\subsection{Grain Sizes}

There exists considerable uncertainty regarding the sizes of grains in the expanding shells. Papoular \& Pegourie (1983) find that these grains might be larger than interstellar grains and Snow et al. (1987) suggest that they are not smaller than 0.05 $\mu \mathrm{m}$, and could be as large as $0.3 \mu \mathrm{m}$. Our results, utilizing compilations by Draine (1987) for optical properties of astronomical silicates and graphites, enable us to draw some conclusions about the grain size distribution. Figure 1 displays the terminal velocities of the gas and the dust along with their difference, the drift velocity, as functions of grain size for model oxygen and carbon stars. In each case the calculations were carried out both with and without scattering opacity. The figure shows that, for $a \lesssim 0.1 \mu \mathrm{m}$, scattering is negligible, the results are essentially independent of the grain size (cf. $\S 4.1$ ), and the gas terminal velocity that we get is comparable with observed values. Larger grains $(0.3-1 \mu \mathrm{m})$ lead to higher outflow and drift velocities. When scattering is ignored, the outflow velocity is still compatible with observed values. But 

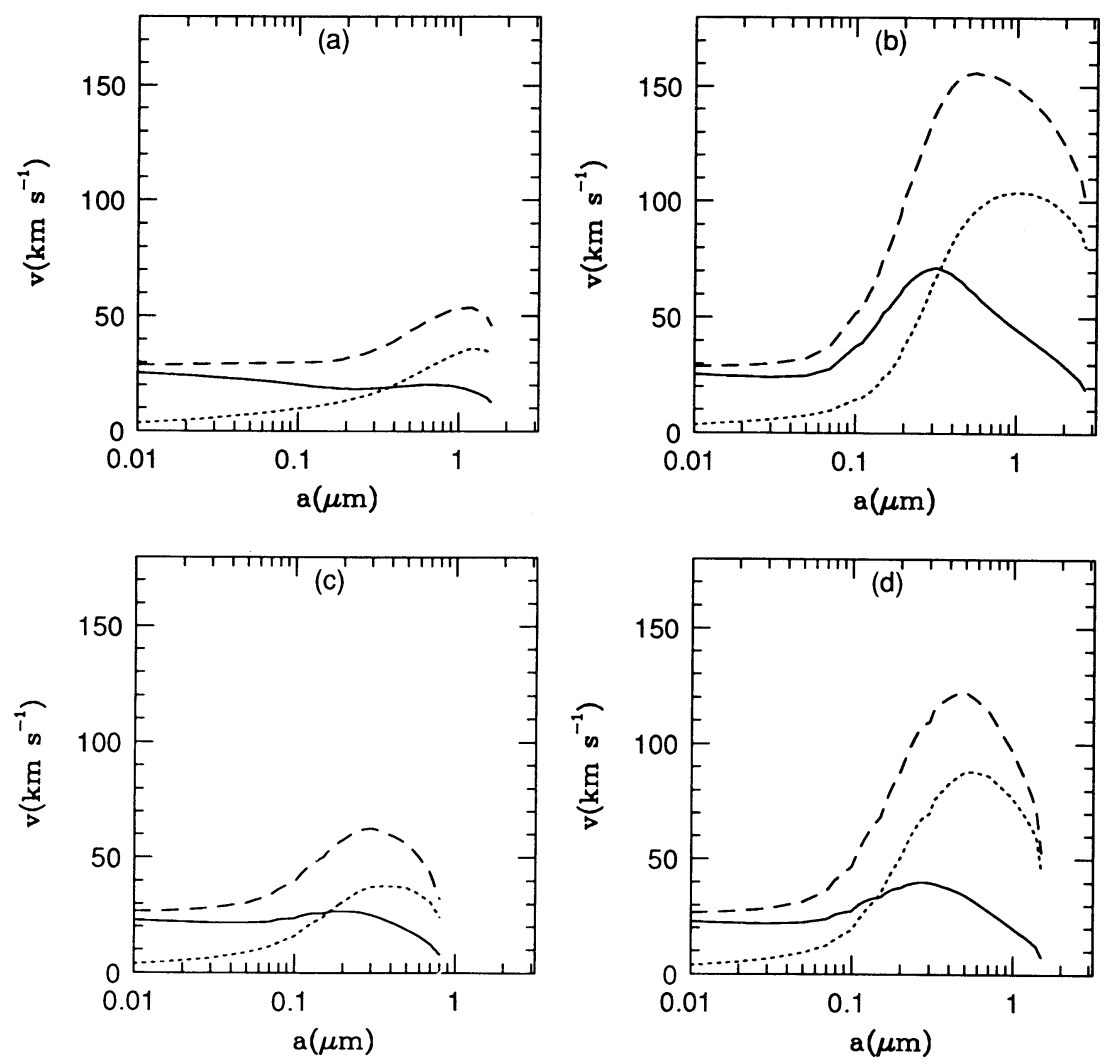

Fig. 1.- Terminal gas outflow velocity (solid lines), dust velocity (dashed lines), and drift velocity (dotted lines) as functions of grain size for (a) an oxygen star neglecting dust scattering opacity, $(b)$ an oxygen star with scattering, $(c)$ a carbon star without scattering, and $(d)$ a carbon star with scattering. The oxygen star model (panels [a] and [b]) assumes silicate grains, $\dot{M}=1 \times 10^{-5} M_{\odot} \mathrm{yr}^{-1}, L_{*}=5 \times 10^{4} L_{\odot}, M_{*}=3 M_{\odot}$ and an effective temperature of $2500 \mathrm{~K}$. The carbon star model (panels $[c]$ and $[d]$ ) assumes graphite grains, the same mass and mass-loss rate, luminosity of $5.6 \times 10^{4} L_{\odot}$ (based on that of IRC +10216 ; cf. Le Bertre 1987), and temperature of $2000 \mathrm{~K}$.

the inclusion of scattering, which is important, leads to unreasonably high outflow velocities, so these grain sizes must be excluded. When the grains are even larger $(a \gtrsim 1 \mu \mathrm{m})$, the terminal velocity decreases again toward values compatible with observations. Although the possible existence of such grains cannot be ruled out from these results, it is certain that they cannot dominate the optical properties of the dust. The reason is that the peak observed at $9.7 \mu \mathrm{m}$ in emission or absorption in red-giant IR spectra is not produced in such a case. These conclusions remain valid for forward-dominated anisotropic scattering since momentum transfer by scattering from radiation to dust is less efficient in this case.

The drift effect and its dependence on grain size can be understood in terms of equation (35). The second term on the right is of no interest here since it appears also in the equation of motion of the gas and hence does not affect the relative motion of the two components. For very small grains the first term is independent of $a$ while the third term, the dust-gas coupling term, behaves like $1 / a$. Therefore the dust velocity grows with grain size. As the dipole approximation breaks down, scattering becomes important and acceleration by radiation pressure increases significantly. When the grains reach a very large size ( $\gtrsim 1 \mu \mathrm{m}$ ), both $Q_{a}$ and $Q_{s}$ are assumed constant and both the first and third terms decrease like $1 / a$. Then the drift velocity, and also the gas outflow velocity, again decreases.

From these results it follows that grain sizes are mostly of order $0.05 \mu \mathrm{m}$. Larger grains, though possible in principle, would drift very fast, reducing their space density. In addition, at such high drift velocities the grains could suffer significant sputtering (Draine \& Salpeter 1979), although the implications of this effect in this case need further study. Therefore, for simplicity we assume identical size of $0.05 \mu \mathrm{m}$ for the grains. With this assumption we find that mass outflow in red giants driven by radiation pressure on dust will always have a lower limit on $\dot{M}$. This limit is best illustrated by Figure 2, which presents the terminal outflow velocity versus mass-loss rate for oxygen and carbon stars. The stars used in this comparison between theory and observation are listed in Table 1 .

As the mass-loss rate decreases toward $\sim 10^{-7} M_{\odot} \mathrm{yr}^{-1}$, the outflow velocity decreases to the thermal velocity. At lower mass-loss rates, the coupling of gas and dust is so weak that the kinetic energy transferred to the gas is insufficient to overcome gravity. This effect may have been observed by Hagen, Stencel, \& Dickinson (1983), who conclude on the basis of observations of red giants and supergiants that radiation pressure cannot be responsible for the mass-loss. In fact, their analysis is based on stars which, with almost no exception, are below the limit we derive and in these stars the envelope expansion indeed must be driven by another mechanism; it should also be noted that the expansion velocities in their sample are rather low $(\lesssim 7 \mathrm{~km}$ $\mathrm{s}^{-1}$ ). Conceivably, the mechanism responsible for lifting the material to the dust formation point is capable of providing it with a small velocity, sufficient to overcome gravity. Higher velocity is then possible only at higher mass-loss rates due to radiation pressure on dust. 

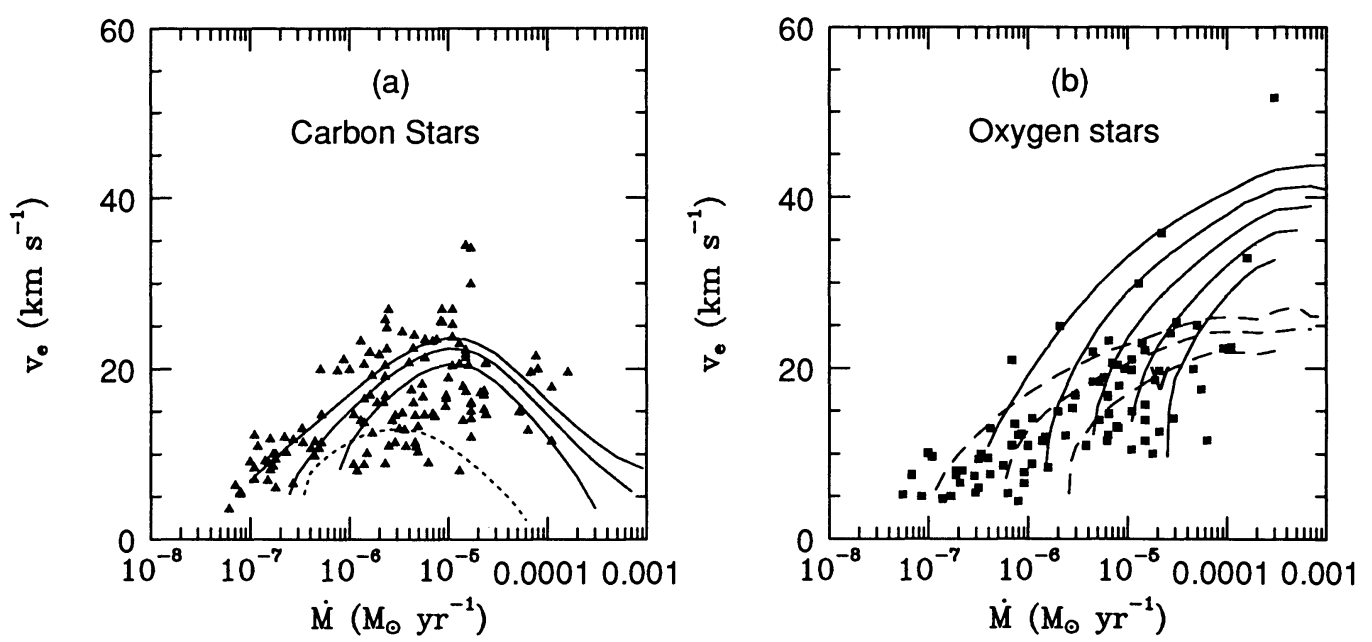

Fig. 2.-Terminal outflow velocity vs. mass-loss rate for calculated models and individual stars. Curves present the results of calculations while individual data points are from Knapp (1991; see Table 1 for detailed listing). (a) Carbon stars with $T_{\text {eff }}=2000 \mathrm{~K}$, assuming a grain size of $0.05 \mu \mathrm{m}$. The solid curves correspond, from top to bottom, to stellar masses of $1.5,3$, and $5 M_{\odot}$ and a luminosity of $5.6 \times 10^{4} L_{\odot}$. The dashed curve corresponds to a star with a mass of $1 M_{\odot}$ and a luminosity of $1 \times 10^{4} L_{\odot}$. (b) Oxygen stars with $T_{\text {eff }}=2500 \mathrm{~K}$ and a grain size of $0.05 \mu \mathrm{m}$. Dashed curves correspond, from top to bottom, to stellar masses of $1.5,3$, and $5 M_{\odot}$ and a luminosity of $5 \times 10^{4} L_{\odot}$. Solid curves correspond, from top to bottom, to supergiants with masses of $10,20,30,40$, and $50 M_{\odot}$, and a luminosity of $4 \times 10^{5} L_{\odot}$.

\subsection{Dust Optical Properties and the Dynamics}

In order to discuss the implications of the dust optical properties on the dynamical problem, consider first the simple case where the dust is assumed to have a gray opacity and the drift effect is ignored. Then the spectral distribution of the radiation is independent of radius, as is the mean opacity $\chi_{F}$. Equation (6) can then be solved at once and the result is the familiar expression (e.g., Goldreich \& Scoville 1976)

$$
v^{2}(r)=v^{2}\left(R_{0}\right)+\frac{\chi_{F} L_{*}}{2 \pi R_{0} c}\left(1-\frac{R_{0}}{r}\right),
$$

which shows that the velocity profile is independent of the mass-loss rate; as already mentioned, our numerical scheme properly reproduces this solution. The initial outflow velocity, $v\left(R_{0}\right)$, is taken at the sonic point (cf. Deguchi 1980). Since it is much smaller than the terminal velocity, the implication of its exact value for the solution is negligible; this is also true for nongray dust, and for the case that the drift effect is included. However, as the mass-loss rate is increased, the diffuse radiation inside the cavity becomes important, $R_{0}$ is pushed farther out, and the terminal velocity (inversely proportional to $R_{0}$ ) is reduced. We have verified that when a gray opacity is used in our numerical scheme it properly reproduces this solution.

For the actual case of nongray dust, the behavior of $\chi_{F}$ as a function of radius must be known. From equations (5) and (13),

$$
\frac{d \chi_{F}}{d r}=\frac{\dot{M}}{L_{*} v} \int \chi_{v} \chi_{v}^{a}\left[4 \pi B_{v}(T)-c u_{v}\right] d v+\chi_{F} \frac{d}{d r} \ln \frac{v}{v_{d}} .
$$

The second term on the right is always positive. The first term, on the other hand, is negative when $\chi_{v}$ is a monotonically increasing function of $v$ and positive in the opposite case. The reason is the following: In the inner zones $u_{v}$ is dominated by the stellar radiation, which can be approximated by a diluted blackbody field at a temperature of $\sim 2000-3000 \mathrm{~K}$, much higher than the dust temperature. As a result, the integrand is dominated by its first term at low frequencies and the second term at high frequencies. Without the factor $\chi_{v}$ the integral vanishes because the equilibrium condition states that $\int \chi_{v}^{a}\left[4 \pi B_{v}(T)-c u_{v}\right] d v=0$. The weighting with a function that emphasizes different frequency regions then causes the integral to be positive or negative, as stated above.

In carbon stars, $\chi_{v}$ is a monotonically increasing function of $v$ and the integral on the right-hand side of equation (39) is negative. In oxygen stars, on the other hand, the opposite is true and $\chi_{F}(r)$ is a monotonically increasing function of radius (since both terms on the right are always positive). In addition, since the first term on the right is proportional to $\dot{M}$, it becomes negligible at small mass-loss rates. In such cases, $\chi_{F}(r)$ is a monotonically increasing function of $r$ for all stars, both oxygen-rich and carbon-rich. As the mass-loss rate increases, the first term dominates and $\chi_{F}$ tends to become a decreasing function of $r$ for carbon stars with large mass-loss rates.

This discussion enables us to reach certain qualitative conclusions concerning the behavior of the solutions. In thin envelopes (small $\dot{M}$ ) the first term on the right-hand side of equation (39) can be neglected, leading to the result

$$
\chi_{F}\left(1+\frac{v_{\mathrm{drift}}}{v}\right)=\text { const }
$$

As shown earlier ( $(2)$, for a given opacity $v$ is independent of $\dot{M}$ while $v_{\text {drift }}$ increases as $\dot{M}$ decreases. Thus $\chi_{F}$ decreases with $\dot{M}$, setting a lower limit on the possible mass-loss rates that can be driven by radiation pressure on dust. This is evident from Figure 2 , which shows the expansion velocity as function of mass-loss rate. Similar lower bounds were obtained by Kwok (1975), and Table 2 presents a comparison between the two sets of results. The limits we find are lower than his, typically by about factor of 4 , although in one case (corresponding to the highest mass and $T_{\text {eff }}$ ) the difference is as large as factor of 10 . These differences can be attributed to the detailed, updated optical properties we employ, as well as the improved treatment of radiative equilibrium. In particular, we find that grains reach their final size at about 3-4 stellar radii, and that another mechanism must be responsible for lifting the material to that distance.

For optically thick envelopes (high mass-loss rates) the behavior of the opacity depends on the composition of the 
TABLE 1

Mass-Loss Rates and Terminal Outflow Velocities of the Stars ApPearing in Figure 2

\begin{tabular}{|c|c|c|c|c|c|c|c|}
\hline Star & $\begin{array}{c}\dot{M} \\
\left(M_{\odot} \mathrm{yr}^{-1}\right)\end{array}$ & $\begin{array}{c}v_{e} \\
\left(\mathrm{~km} \mathrm{~s}^{-1}\right)\end{array}$ & Reference & Star & $\begin{array}{c}\dot{M} \\
\left(M_{\odot} \mathrm{yr}^{-1}\right)\end{array}$ & $\begin{array}{c}v_{e} \\
\left(\mathrm{~km} \mathrm{~s}^{-1}\right)\end{array}$ & Reference \\
\hline \multicolumn{4}{|c|}{ Oxygen Stars } & \multicolumn{4}{|c|}{ Oxygen Stars } \\
\hline IRC $+40004 \ldots \ldots \ldots \ldots \ldots \ldots$ & $2.7 \times 10^{-5}$ & 24.2 & 1 & IRC -10529 . & $1.5 \times 10^{-5}$ & 15.8 & 1 \\
\hline T Cas $\ldots \ldots \ldots \ldots \ldots \ldots \ldots \ldots$ & $5.6 \times 10^{-8}$ & 5.2 & 2 & IRC $+80040 \ldots \ldots \ldots \ldots \ldots \ldots$ & $1.5 \times 10^{-6}$ & 12.0 & 11 \\
\hline IRC $+10011 \ldots \ldots \ldots \ldots \ldots \ldots$ & $1.4 \times 10^{-5}$ & 23.0 & 1 & CRL $2646 \ldots \ldots \ldots \ldots \ldots \ldots$ & $2.8 \times 10^{-6}$ & 15.4 & 6 \\
\hline IRC $+30021 \ldots \ldots \ldots \ldots \ldots \ldots$ & $8.0 \times 10^{-6}$ & 13.0 & 3 & T Cep $\ldots \ldots \ldots \ldots \ldots \ldots \ldots$ & $8.6 \times 10^{-8}$ & 5.0 & 6 \\
\hline CRL $278 \ldots \ldots \ldots \ldots \ldots \ldots \ldots$ & $4.2 \times 10^{-7}$ & 7.6 & 2 & IRC $+40483 \ldots \ldots \ldots \ldots \ldots \ldots$ & $8.2 \times 10^{-6}$ & 18.0 & 1 \\
\hline$o$ Cet $\ldots \ldots \ldots \ldots \ldots \ldots \ldots \ldots$ & $1.7 \times 10^{-7}$ & 5.0 & 1 & UU Peg $\ldots \ldots \ldots \ldots \ldots \ldots \ldots \ldots$ & $7.4 \times 10^{-7}$ & 13.5 & 6 \\
\hline CIT $4 \ldots \ldots \ldots$ & $6.4 \times 10^{-6}$ & 12.2 & 3 & RU Cyg $\ldots \ldots \ldots \ldots \ldots \ldots \ldots$ & $1.1 \times 10^{-6}$ & 14.2 & 6 \\
\hline IRC $-30023 \ldots \ldots \ldots \ldots \ldots$ & $1.4 \times 10^{-6}$ & 11.6 & 2 & $\mu$ Cep $\ldots \ldots \ldots \ldots \ldots \ldots \ldots \ldots$ & $3.4 \times 10^{-7}$ & 10.0 & 12 \\
\hline R Hor $\ldots \ldots \ldots \ldots \ldots \ldots \ldots$ & $2.1 \times 10^{-7}$ & 6.6 & 4 & EP Aqr $\ldots \ldots \ldots \ldots \ldots \ldots \ldots$ & $5.7 \times 10^{-7}$ & 8.6 & 2 \\
\hline NML Tau $\ldots \ldots \ldots \ldots \ldots \ldots$ & $4.5 \times 10^{-6}$ & 22.0 & 1 & RT Cep $\ldots \ldots \ldots \ldots \ldots \ldots \ldots$ & $4.5 \times 10^{-6}$ & 18.5 & 11 \\
\hline V Eri $\ldots \ldots \ldots \ldots \ldots \ldots \ldots \ldots$ & $4.2 \times 10^{-7}$ & 13.0 & 2 & IRAS $2155+6204 \ldots \ldots \ldots \ldots$ & $2.1 \times 10^{-5}$ & 12.6 & 2 \\
\hline TX Cam $\ldots \ldots \ldots \ldots \ldots \ldots \ldots$ & $3.0 \times 10^{-6}$ & 16.9 & 5 & TW Peg $\ldots \ldots \ldots \ldots \ldots \ldots \ldots$ & $4.0 \times 10^{-7}$ & 9.5 & 2 \\
\hline IRC $+50137 \ldots \ldots \ldots \ldots \ldots \ldots$ & $6.5 \times 10^{-6}$ & 14.7 & 1 & SV Peg $\ldots \ldots \ldots \ldots \ldots \ldots \ldots$ & $1.0 \times 10^{-6}$ & 11.0 & 10 \\
\hline R Aur $\ldots \ldots \ldots \ldots \ldots$ & $1.0 \times 10^{-6}$ & 10.9 & 1 & IRAS $2227+5435 \ldots \ldots \ldots \ldots$ & $6.3 \times 10^{-5}$ & 11.6 & 3 \\
\hline $\mathrm{IRC}+60154 \ldots \ldots \ldots \ldots \ldots \ldots$ & $1.1 \times 10^{-5}$ & 19.9 & 2 & V PsA $\ldots \ldots \ldots \ldots \ldots \ldots \ldots \ldots$ & $6.9 \times 10^{-7}$ & 21.0 & 4 \\
\hline IRC $+70066 \ldots \ldots \ldots \ldots \ldots \ldots$ & $1.1 \times 10^{-5}$ & 21.1 & 1 & CRL 2999 .................. & $5.5 \times 10^{-5}$ & 17.6 & 10 \\
\hline V Cam $\ldots \ldots \ldots \ldots \ldots \ldots \ldots$ & $8.0 \times 10^{-7}$ & 12.2 & 6 & IRS $+60427 \ldots .$. & $5.8 \times 10^{-6}$ & 19.0 & 4 \\
\hline IRC $+40149 \ldots \ldots \ldots \ldots \ldots$ & $2.1 \times 10^{-5}$ & 19.8 & 7 & $\mathrm{R}$ Cas...$\ldots \ldots \ldots \ldots \ldots \ldots \ldots \ldots$ & $8.6 \times 10^{-7}$ & 12.3 & 1 \\
\hline IRC $+60169 \ldots \ldots \ldots \ldots \ldots \ldots$ & $6.3 \times 10^{-6}$ & 16.9 & 1 & & & & \\
\hline IRC $20101 \ldots \ldots \ldots \ldots \ldots \ldots$ & $9.4 \times 10^{-6}$ & 20.0 & 8 & \multirow{2}{*}{\multicolumn{4}{|c|}{ Carbon Stars }} \\
\hline GX Mon .................... & $1.9 \times 10^{-5}$ & 18.7 & 2 & & & & \\
\hline IRAS $0713+1005 \ldots \ldots \ldots \ldots$ & $1.8 \times 10^{-5}$ & 10.0 & 3 & IRAS $0021+6221 \ldots \ldots \ldots \ldots$ & $3.2 \times 10^{-6}$ & 13.0 & 13 \\
\hline VY CMa $\ldots \ldots \ldots \ldots \ldots \ldots \ldots$ & $2.2 \times 10^{-5}$ & 35.9 & 2 & IRC $+60041 \ldots \ldots \ldots \ldots \ldots \ldots$ & $1.3 \times 10^{-6}$ & 23.3 & 6 \\
\hline Y Lyn ...................... & $3.0 \times 10^{-7}$ & 5.4 & 5 & Z Psc $\ldots \ldots \ldots \ldots \ldots \ldots \ldots \ldots$ & $6.1 \times 10^{-8}$ & 3.5 & 14 \\
\hline RS Cnc $\ldots \ldots \ldots \ldots \ldots \ldots \ldots$ & $6.3 \times 10^{-7}$ & 5.3 & 1 & CRL $190 \ldots \ldots \ldots \ldots \ldots \ldots$ & $6.8 \times 10^{-5}$ & 19.7 & 2 \\
\hline IRAS $0937+1212 \ldots \ldots \ldots \ldots$ & $2.1 \times 10^{-6}$ & 25.0 & 8 & R Scl $\ldots \ldots \ldots \ldots \ldots \ldots \ldots \ldots$ & $4.2 \times 10^{-6}$ & 17.6 & 15 \\
\hline R LMi ..................... & $3.2 \times 10^{-7}$ & 6.0 & 9 & IRAS $0215+2822 \ldots \ldots \ldots \ldots$ & $2.3 \times 10^{-6}$ & 8.9 & 6 \\
\hline IW Hyа...$\ldots \ldots \ldots \ldots \ldots \ldots$ & $5.2 \times 10^{-6}$ & 14.0 & 2 & R For $\ldots \ldots \ldots \ldots \ldots \ldots$ & $1.0 \times 10^{-6}$ & 20.0 & 15 \\
\hline R Leo $\ldots \ldots \ldots \ldots \ldots \ldots \ldots \ldots \ldots$ & $9.2 \times 10^{-7}$ & 6.5 & 9 & CRL $341 \ldots \ldots \ldots \ldots \ldots \ldots \ldots$ & $1.7 \times 10^{-5}$ & 14.2 & 3 \\
\hline $\mathrm{R} \mathrm{Crt} \ldots \ldots \ldots \ldots \ldots$ & $6.9 \times 10^{-7}$ & 11.0 & 2 & TW Hor $\ldots \ldots \ldots \ldots \ldots \ldots \ldots$ & $8.0 \times 10^{-8}$ & 5.3 & 15 \\
\hline IRC $-30163 E \ldots \ldots \ldots \ldots \ldots$ & $2.2 \times 10^{-7}$ & 8.0 & 2 & CRL $482 \ldots \ldots \ldots \ldots \ldots \ldots$ & $1.7 \times 10^{-5}$ & 16.0 & 1 \\
\hline BK Vir $\ldots \ldots \ldots \ldots \ldots \ldots \ldots$ & $1.4 \times 10^{-7}$ & 4.7 & 6 & IRC $+50096 \ldots \ldots \ldots \ldots \ldots \ldots$ & $5.5 \times 10^{-6}$ & 16.8 & 1 \\
\hline RT Vir $\ldots \ldots \ldots \ldots \ldots \ldots \ldots$ & $1.6 \times 10^{-6}$ & 8.4 & 10 & U Cam ...................... & $1.6 \times 10^{-6}$ & 22.0 & 3 \\
\hline SW Vir $\ldots \ldots \ldots \ldots \ldots \ldots \ldots \ldots$ & $3.2 \times 10^{-7}$ & 9.4 & 11 & CRL $5102 \ldots \ldots \ldots \ldots \ldots \ldots$ & $4.9 \times 10^{-6}$ & 13.3 & 2 \\
\hline R Hya $\ldots \ldots \ldots \ldots \ldots \ldots \ldots \ldots$ & $6.9 \times 10^{-8}$ & 7.5 & 5 & IRC $+60144 \ldots \ldots \ldots \ldots \ldots \ldots$ & $1.1 \times 10^{-5}$ & 20.4 & 1 \\
\hline 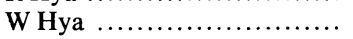 & $1.1 \times 10^{-7}$ & 9.7 & 5 & CRL $618 \ldots \ldots \ldots \ldots \ldots \ldots \ldots$ & $7.7 \times 10^{-5}$ & 21.5 & 1 \\
\hline RX Boo $\ldots \ldots \ldots \ldots \ldots \ldots \ldots$ & $9.2 \times 10^{-7}$ & 7.8 & 9 & 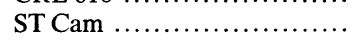 & $1.8 \times 10^{-7}$ & 10.0 & 14 \\
\hline WX Ser $\ldots \ldots \ldots \ldots \ldots \ldots \ldots$ & $1.4 \times 10^{-6}$ & 11.5 & 7 & IRAS $0453+4427 \ldots \ldots \ldots \ldots$ & $4.0 \times 10^{-6}$ & 20.8 & 16 \\
\hline X Her $\ldots \ldots \ldots \ldots \ldots \ldots \ldots \ldots$ & $1.1 \times 10^{-6}$ & 8.8 & 2 & R Lep $\ldots \ldots \ldots \ldots \ldots \ldots \ldots \ldots$ & $4.5 \times 10^{-7}$ & 9.8 & 2 \\
\hline CRL $1822 \ldots \ldots \ldots \ldots \ldots \ldots$ & $2.9 \times 10^{-5}$ & 14.2 & 4 & W Ori $\ldots \ldots \ldots \ldots \ldots \ldots \ldots \ldots \ldots$ & $1.2 \times 10^{-7}$ & 10.9 & 17 \\
\hline IRAS $1610-4205 \ldots \ldots \ldots \ldots$ & $1.5 \times 10^{-5}$ & 14.0 & 10 & IRAS $0510+2055 \ldots \ldots \ldots \ldots$ & $1.1 \times 10^{-5}$ & 25.2 & 16 \\
\hline $30 \mathrm{~g}$ Her $\ldots \ldots \ldots \ldots \ldots \ldots \ldots$ & $1.9 \times 10^{-7}$ & 7.5 & 2 & IRAS $0513+4712 \ldots \ldots \ldots \ldots$ & $5.7 \times 10^{-6}$ & 14.7 & 16 \\
\hline NGC $6302 \ldots \ldots \ldots \ldots \ldots \ldots$ & $1.1 \times 10^{-4}$ & 22.5 & 6 & 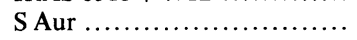 & $1.4 \times 10^{-6}$ & 16.5 & 3 \\
\hline CRL 6815S $\ldots \ldots \ldots \ldots \ldots \ldots$ & $5.0 \times 10^{-5}$ & 25.1 & 10 & CRL $809 \ldots \ldots \ldots \ldots \ldots \ldots \ldots$ & $1.7 \times 10^{-5}$ & 30.0 & 7 \\
\hline MW Her...$\ldots \ldots \ldots \ldots \ldots$ & $6.5 \times 10^{-6}$ & 23.3 & 3 & W Pic $\ldots \ldots \ldots \ldots \ldots \ldots \ldots$ & $1.1 \times 10^{-7}$ & 7.0 & 15 \\
\hline CRL $5379 \ldots \ldots \ldots \ldots \ldots \ldots \ldots$ & $7.9 \times 10^{-6}$ & 20.5 & 10 & Y Tau $\ldots \ldots \ldots \ldots \ldots \ldots \ldots \ldots$ & $4.1 \times 10^{-7}$ & 10.7 & 15 \\
\hline $161796 \ldots \ldots \ldots \ldots \ldots \ldots \ldots$ & $1.5 \times 10^{-5}$ & 11.5 & 8 & CRL $865 \ldots \ldots \ldots \ldots \ldots \ldots$ & $2.3 \times 10^{-5}$ & 15.3 & 1 \\
\hline 89 Her $\ldots \ldots \ldots \ldots \ldots \ldots \ldots \ldots$ & $8.0 \times 10^{-7}$ & 4.4 & 8 & TU Gem $\ldots \ldots \ldots \ldots \ldots \ldots \ldots$ & $5.2 \times 10^{-7}$ & 11.4 & 14 \\
\hline VX Sag $\ldots \ldots \ldots \ldots \ldots \ldots \ldots \ldots$ & $1.3 \times 10^{-5}$ & 30.0 & 4 & CRL $933 \ldots \ldots \ldots \ldots \ldots \ldots$ & $2.3 \times 10^{-6}$ & 25.8 & 6 \\
\hline IRC $+10365 \ldots \ldots \ldots \ldots \ldots \ldots$ & $6.3 \times 10^{-6}$ & 16.7 & 1 & CRL $935 \ldots \ldots \ldots \ldots \ldots \ldots$ & $3.6 \times 10^{-6}$ & 12.9 & 3 \\
\hline IRC $+10374 \ldots \ldots \ldots \ldots \ldots$ & $5.2 \times 10^{-6}$ & 18.5 & 3 & CRL $954 \ldots \ldots \ldots \ldots \ldots \ldots \ldots$ & $5.8 \times 10^{-6}$ & 21.4 & 3 \\
\hline IRAS $1846-4802 \ldots \ldots \ldots \ldots$ & $3.8 \times 10^{-6}$ & 11.0 & 4 & UU Aur.......$\ldots \ldots \ldots \ldots$ & $4.5 \times 10^{-7}$ & 11.5 & 2 \\
\hline IRC $30398 \ldots \ldots \ldots \ldots \ldots \ldots$ & $7.7 \times 10^{-6}$ & 13.2 & 10 & CRL $971 \ldots \ldots \ldots \ldots \ldots \ldots$ & $6.3 \times 10^{-6}$ & 9.0 & 2 \\
\hline RS Cra $\ldots \ldots \ldots \ldots \ldots \ldots \ldots$ & $7.0 \times 10^{-6}$ & 20.7 & 10 & CL Mon $\ldots \ldots \ldots \ldots \ldots \ldots$ & $2.5 \times 10^{-6}$ & 27.0 & 6 \\
\hline R Aql $\ldots \ldots \ldots \ldots \ldots \ldots \ldots \ldots \ldots$ & $1.0 \times 10^{-7}$ & 10.1 & 5 & W CMa $\ldots \ldots \ldots \ldots \ldots \ldots$ & $2.7 \times 10^{-7}$ & 11.7 & 15 \\
\hline 3880 Sag $\ldots \ldots \ldots \ldots \ldots \ldots \ldots$ & $1.5 \times 10^{-5}$ & 22.2 & 10 & 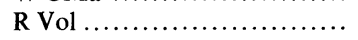 & $1.7 \times 10^{-6}$ & 19.3 & 15 \\
\hline IRC $30404 \ldots \ldots \ldots \ldots \ldots \ldots$ & $1.1 \times 10^{-5}$ & 15.0 & 4 & NGC $2346 \ldots \ldots \ldots \ldots \ldots \ldots$ & $1.0 \times 10^{-5}$ & 19.0 & 7 \\
\hline CRL $2343 \ldots \ldots \ldots \ldots \ldots \ldots$ & $1.6 \times 10^{-4}$ & 33.0 & 8 & CRL $1085 \ldots \ldots \ldots \ldots \ldots \ldots$ & $1.1 \times 10^{-5}$ & 23.8 & 2 \\
\hline CRL $2362 \ldots \ldots \ldots \ldots \ldots \ldots$ & $4.6 \times 10^{-5}$ & 20.0 & 10 & IRAS $0721-1246 \ldots \ldots \ldots \ldots$ & $2.4 \times 10^{-6}$ & 24.9 & 16 \\
\hline Vy $2-2 \ldots \ldots \ldots \ldots \ldots \ldots \ldots \ldots$ & $2.4 \times 10^{-6}$ & 12.1 & 1 & IRC $-20131 \ldots \ldots \ldots \ldots \ldots \ldots$ & $8.7 \times 10^{-6}$ & 25.5 & 6 \\
\hline IRC $+10420 \ldots \ldots \ldots \ldots \ldots$ & $3.0 \times 10^{-4}$ & 51.7 & 1 & IRAS $0758-1933 \ldots \ldots \ldots \ldots$ & $4.6 \times 10^{-6}$ & 14.6 & 16 \\
\hline M1 $-92 \ldots \ldots \ldots \ldots \ldots \ldots$ & $3.1 \times 10^{-5}$ & 25.5 & 7 & IRAS $0804-1524 \ldots \ldots \ldots \ldots$ & $1.4 \times 10^{-6}$ & 13.7 & 16 \\
\hline GY Aql $\ldots \ldots \ldots \ldots \ldots \ldots \ldots$ & $6.2 \times 10^{-6}$ & 11.5 & 5 & IRAS $0807-3615 \ldots \ldots \ldots \ldots$ & $2.1 \times 10^{-5}$ & 17.3 & 10 \\
\hline CRL $2477 \ldots \ldots \ldots \ldots \ldots \ldots$ & $9.2 \times 10^{-5}$ & 22.4 & 6 & CRL $1235 \ldots \ldots \ldots \ldots \ldots \ldots$ & $2.4 \times 10^{-5}$ & 20.7 & 2 \\
\hline RR Aql $\ldots \ldots \ldots \ldots \ldots \ldots \ldots$ & $2.9 \times 10^{-7}$ & 7.4 & 2 & CRL $5250 \ldots \ldots \ldots \ldots \ldots \ldots$ & $1.7 \times 10^{-5}$ & 16.1 & 2 \\
\hline IRAS $2002+3910 \ldots \ldots \ldots \ldots$ & $1.1 \times 10^{-5}$ & 10.5 & 2 & $\mathrm{X} \mathrm{Cnc} \ldots \ldots \ldots \ldots \ldots \ldots \ldots \ldots$ & $2.2 \times 10^{-7}$ & 12.0 & 14 \\
\hline $1943 \mathrm{Sag} \ldots \ldots \ldots \ldots \ldots \ldots \ldots$ & $1.9 \times 10^{-7}$ & 8.0 & 4 & CRL $5254 \ldots \ldots \ldots \ldots \ldots \ldots$ & $6.4 \times 10^{-5}$ & 12.8 & 2 \\
\hline
\end{tabular}


TABLE 1-Continued

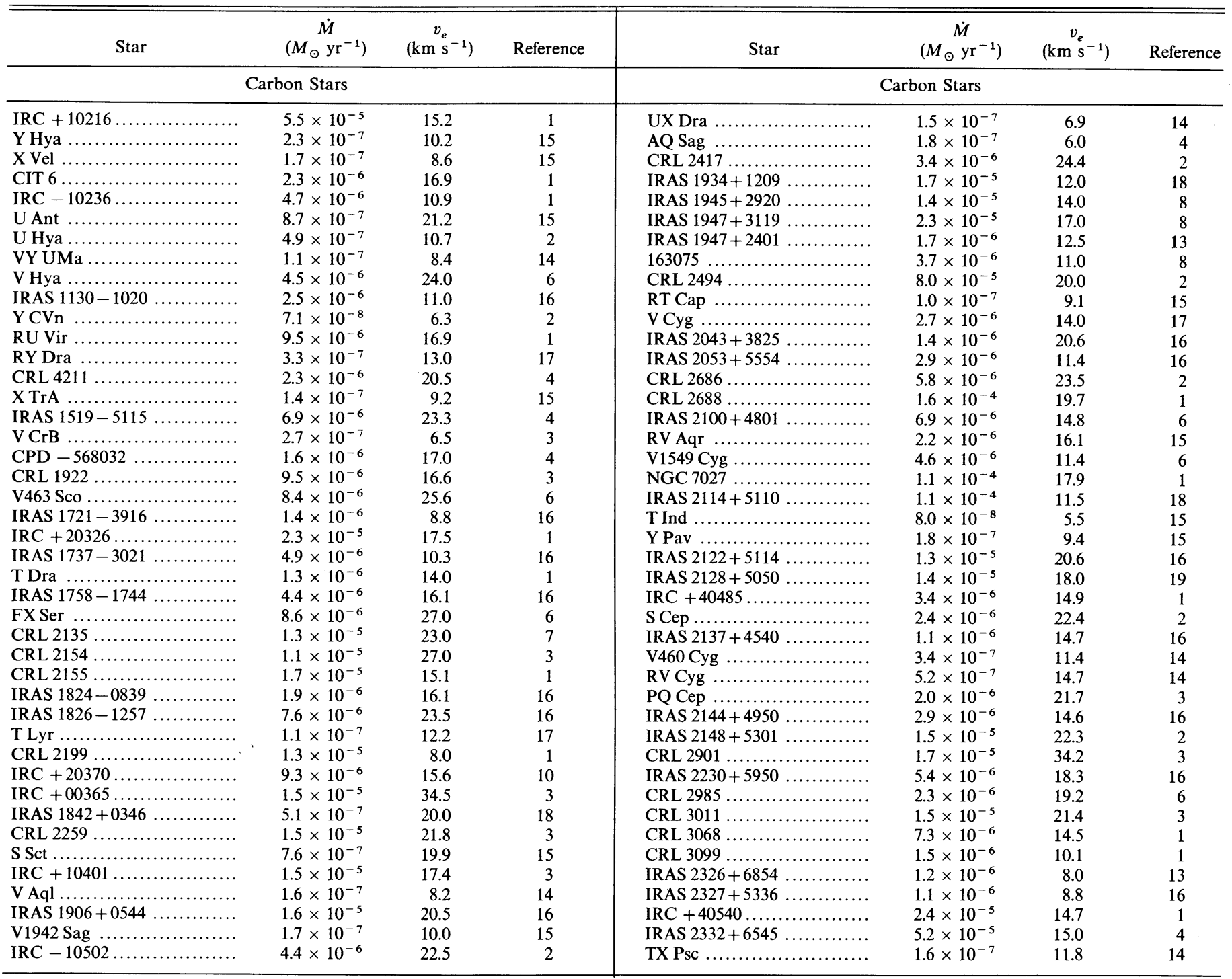

REFERENCES. - (1) Knapp \& Morris 1985; (2) Zuckerman \& Dyck 1986a; (3) Zuckerman, Dyck, \& Claussen 1986; (4) Knapp et al. 1989; (5) Wannier \& Sahai 1986; (6) Zuckerman \& Dyck 1989; (7) Knapp 1986; (8) Likkel et al. 1987; (9) Knapp et al. 1982; (10) Zuckerman \& Dyck 1986b; (11) G. Knapp (private communication); (12) LeBorgne \& Mauron 1989; (13) Arquilla, Leahy, \& Kwok 1986; (14) Olofsson, Eriksson, \& Gustafsson 1987; (15) Olofsson, Eriksson, \& Gustafsson 1988; (16) Rieu et al. 1987; (17) Jura, Kahane, \& Omont 1988;(18) Leahy, Kwok, \& Arquilla 1987;(19) Likkel et al. 1988.

dust. In carbon stars $\chi_{F}$ decreases outward and the rate of decrease is higher as the mass-loss rate increases. Thus the acceleration becomes progressively weaker as $\dot{M}$ increases and

TABLE 2

Comparison of Minimal Mass-Loss Rates, $\dot{M}_{\min }$ in $M_{\odot} \mathrm{yr}^{-1}$, Obtained IN This Work With Those ObTained BY KWOK (1975)

\begin{tabular}{cccccc}
\hline \hline $\begin{array}{c}\text { Mass } \\
\left(M_{\odot}\right)\end{array}$ & $\begin{array}{c}L_{*} \\
\left(L_{\odot}\right)\end{array}$ & $\begin{array}{c}T_{\text {eff }} \\
(\mathrm{K})\end{array}$ & $\begin{array}{c}\text { Grain Size } \\
(\mu \mathrm{m})\end{array}$ & $\begin{array}{c}\dot{M}_{\min } \\
(\mathrm{Kwok})\end{array}$ & $\begin{array}{c}\dot{M}_{\min } \\
(\text { This Work })\end{array}$ \\
\hline $1 \ldots \ldots \ldots \ldots$ & $10^{4}$ & 2000 & 0.15 & $5.5 \times 10^{-7}$ & $1.5 \times 10^{-7}$ \\
$5 \ldots \ldots \ldots \ldots$ & $10^{5}$ & 2000 & 0.11 & $1.8 \times 10^{-6}$ & $4.0 \times 10^{-7}$ \\
$5 \ldots \ldots \ldots \ldots$ & $10^{5}$ & 3000 & 0.06 & $9.8 \times 10^{-7}$ & $2.0 \times 10^{-7}$ \\
$25 \ldots \ldots \ldots$. & $10^{6}$ & 2000 & 0.08 & $6.0 \times 10^{-6}$ & $1.5 \times 10^{-6}$ \\
$25 \ldots \ldots \ldots$. & $10^{6}$ & 3000 & 0.045 & $3.1 \times 10^{-6}$ & $5.0 \times 10^{-7}$ \\
$25 \ldots \ldots \ldots \ldots$ & $10^{6}$ & 5000 & 0.02 & $1.6 \times 10^{-6}$ & $1.5 \times 10^{-7}$ \\
\hline
\end{tabular}

the terminal velocity decreases until eventually the outflow stops. Hence an upper limit on the mass-loss rate is also set in this case. Its value, as evident from Figure $2 a$, is several times $10^{-4} M_{\odot} \mathrm{yr}^{-1}$.

In oxygen stars the situation is more complicated: $\chi_{F}$ increases outward and its rate of increase increases with $\dot{M}$. Hence the terminal velocity would also be expected to increase with $\dot{M}$. This effect, however, is countered by the diffuse radiation in the cavity that pushes outwards the dust formation point. The combined effect of these two competing phenomena is that for high mass-loss rates the terminal velocity varies very little with $\dot{M}$, can be seen from Figure $2 b$.

Although our calculations did not produce an upper limit on $\dot{M}$ for oxygen stars, such a limit must eventually be reached. This should happen when the envelope becomes so thick that the spectral distribution of the radiation is shifted beyond the 
silicate absorption peaks. In such a case, the behavior of $\chi_{F}$ for oxygen stars should become similar to that for carbon stars, ultimately producing an upper limit on $\dot{M}$. Such a limit, however, would be of little interest since the involved mass-loss rates must be considerably larger than all those considered here, beyond the observed range.

It should be pointed out that when a limit mass-loss rate is approached, the linear relationship between optical depth and mass-loss rate, discussed in $\S 2$, breaks down. The reason is that the terminal velocity then approaches the escape velocity so that gravity can no longer be neglected, leading to a decrease in outflow velocity and an increase in optical depth.

\subsection{Stellar Properties and Their Implications on the Solution}

Some of the parameters characterizing late-type stars with potential significance for the outflow unfortunately involve observational uncertainties, including the bolometric luminosity as well as the spectrum. Even when the star is assumed to emit as blackbody, the radiation still undergoes molecular absorption on its way to the dust shell. Fortunately, the solution is not severely affected by these uncertainties. Regarding the luminosity dependence, Jura (1984) has shown qualitatively that $v_{e} \sim L_{*}^{1 / 4}$, and this moderate dependence is supported by our calculations. Therefore, outflow velocities vary by less than a factor of 2 when $L_{*}$ is varied over the full range of giant and supergiant luminosities, about one order of magnitude. Regarding the stellar spectrum, our calculations show that the velocity profile for a given model is almost independent of the stellar effective temperature. Since little is known about the absorption-line spectrum, we devised a simple method to test its effect: the stellar radiation field is given a blackbody shape, but certain bands are removed at random (assuming that their corresponding secondary radiation escapes at long IR wavelengths). Using this procedure, we found no effect on the dynamics other than that caused by the effective reduction of the stellar luminosity (a small effect, as just mentioned). This leads us to conclude that the absorption lines have a rather minor impact on the problem.

A parameter with important impact on the solution is the dust-to-gas ratio. In our calculations we assumed a ratio by mass of $1.5 \times 10^{-3}$ for all carbon stars and $5 \times 10^{-3}$ for oxygen stars (Knapp 1985). It is quite possible, though, that this ratio could vary within each group. Figure 2 shows that the terminal velocity for oxygen stars is almost independent of $\dot{M}$ at high mass-loss rates, yet observations show that stars with high $\dot{M}$ and lower outflow velocities do exist. A possible explanation is suggested by the results of Baud et al. (1981), who find that $\mathrm{OH} / \mathrm{IR}$ stars with terminal velocities less than 15 $\mathrm{km} \mathrm{s}^{-1}$ tend to belong to old disk population. This implies that a reduced metallicity, and hence a reduced dust-to-gas ratio, are to be expected. Figure 3 displays the impact of various parameters on the profile and terminal velocity. The input parameters of the models along with the resulting outflow velocity, optical depths at 1 and $10 \mu \mathrm{m}$, and flux-mean optical depth are listed in Table 3.

\subsection{The Inner Cavity Diffuse Radiation}

The inherent differences between the solutions for carbon stars and oxygen stars are best demonstrated by the spectral shape of diffuse radiation in the inner cavity (see $\S 3.3$ ), presented in Figure 4. In general, the larger the mass-loss rate, the more efficient is the processing of radiation into longer wavelengths. In carbon stars (Fig. 4a), lowering the mass-loss rate makes the escape of processed radiation easier, and the dust temperature is lowered accordingly. This is why the peak of the spectral distribution shifts to longer wavelengths and the intensity increases as the mass-loss rate increases.

The situation in oxygen stars is more complicated. The secondary radiation is concentrated closer to the silicate absorption peaks, resulting in a greenhouse effect. As a result, the rate of decrease of dust temperature with distance from the star is moderate and almost independent of $\dot{M}$. The IR field in the inner cavity is characterized by a peak between 10 and $20 \mu \mathrm{m}$ (Fig. $4 b$ ). When the mass-loss rate is very high, the distribution approaches a blackbody shape.

\section{GRADUAL ACCELERATION IN SUPERGIANTS}

Maser observations of the circumstellar envelope of VX Sagittarii provide evidence for considerable acceleration at substantial distances from the central star (Chapman \& Cohen 1986). The observed velocity is $\sim 10 \mathrm{~km} \mathrm{~s}^{-1}$ for the $\mathrm{H}_{2} \mathrm{O}$ and $\mathrm{OH}$ main line masers, while for the $1612 \mathrm{MHz} \mathrm{OH}$ maser, located further out by about 80 stellar radii, it is $19 \mathrm{~km} \mathrm{~s}^{-1}$. Subsequent CO observations by Knapp et al. (1989) indicate a terminal outflow velocity of about $30 \mathrm{~km} \mathrm{~s}^{-1}$. The inferred accelerations are considerably higher than what could be expected at such large distances; radiation pressure on promptly formed dust produces most of its acceleration immediately following dust formation, during the initial stages of the wind outflow. Chapman \& Cohen considered the possibility that grain growth due to ice accretion could lead to enhanced radiation pressure at large radii. However, Netzer (1989) argued that grain growth could not explain the observed acceleration and suggested that a radiation pressure gradient might be responsible instead. Since the $\mathrm{CO}$ observed velocity is dominated by the outermost layers of the envelope, which are too dilute for radiative transfer effects to have any appreciable impact, he suggested that this velocity reflects stellar evolutionary effects which cause the outflow velocity to decrease with time. Using different methods, both Netzer and Knapp et al. conclude that the mass-loss rate of VX Sagittarii is $\dot{M} \simeq 2$ $\times 10^{-5} M_{\odot} \mathrm{yr}^{-1}$.

The detailed calculations presented here enable us to examine the different aspects of this problem. The discussion of $\S 4$ shows that the flux mean opacity grows even if grain growth is completely ignored, leading to a more gradual acceleration pattern than assumed in the past (cf. eq. [38]). In stars with low mass-loss rates, the reason is dust drift, while in oxygen stars (and OH/IR stars in particular) with large $\dot{M}$ the effect arises from the optical properties of silicates. The acceleration pattern is conveniently described in terms of the parameter $\epsilon=\left(r / v_{e}\right)\left(d v_{e} / d r\right)$. We find that typically $\epsilon \lesssim 0.1$ at distances of several tens of stellar radii, the location of the $\mathrm{OH}$ maser shell. This value increases with mass loss rate, in agreement with the recent observations of Bowers \& Johnston (1990). In particular, they find that the red supergiants $\mathrm{OH}$ $127.8-00$ and $\mathrm{OH} 26.5+0.6$ have mass-loss rates on the order of $10^{-4} M_{\odot} \mathrm{yr}^{-1}$ and $\epsilon \simeq 0.1$. VX Sagittarii, along with IRC +10420 with $\epsilon \simeq 0.2$, seems to be exceptional. The uniqueness of VX Sagittarii becomes more conspicuous when we note that IRC +10420 is an F8 star, the only known OH/IR star which is not a late-type star (Bowers 1984).

Our calculations could not produce the high value of $\epsilon$ observed in VX Sagittarii at several tens of stellar radii. A possible explanation for these gradients is the following: With a mass-loss rate of $\sim 2 \times 10^{-5} M_{\odot} \mathrm{yr}^{-1}$ and the outflow 

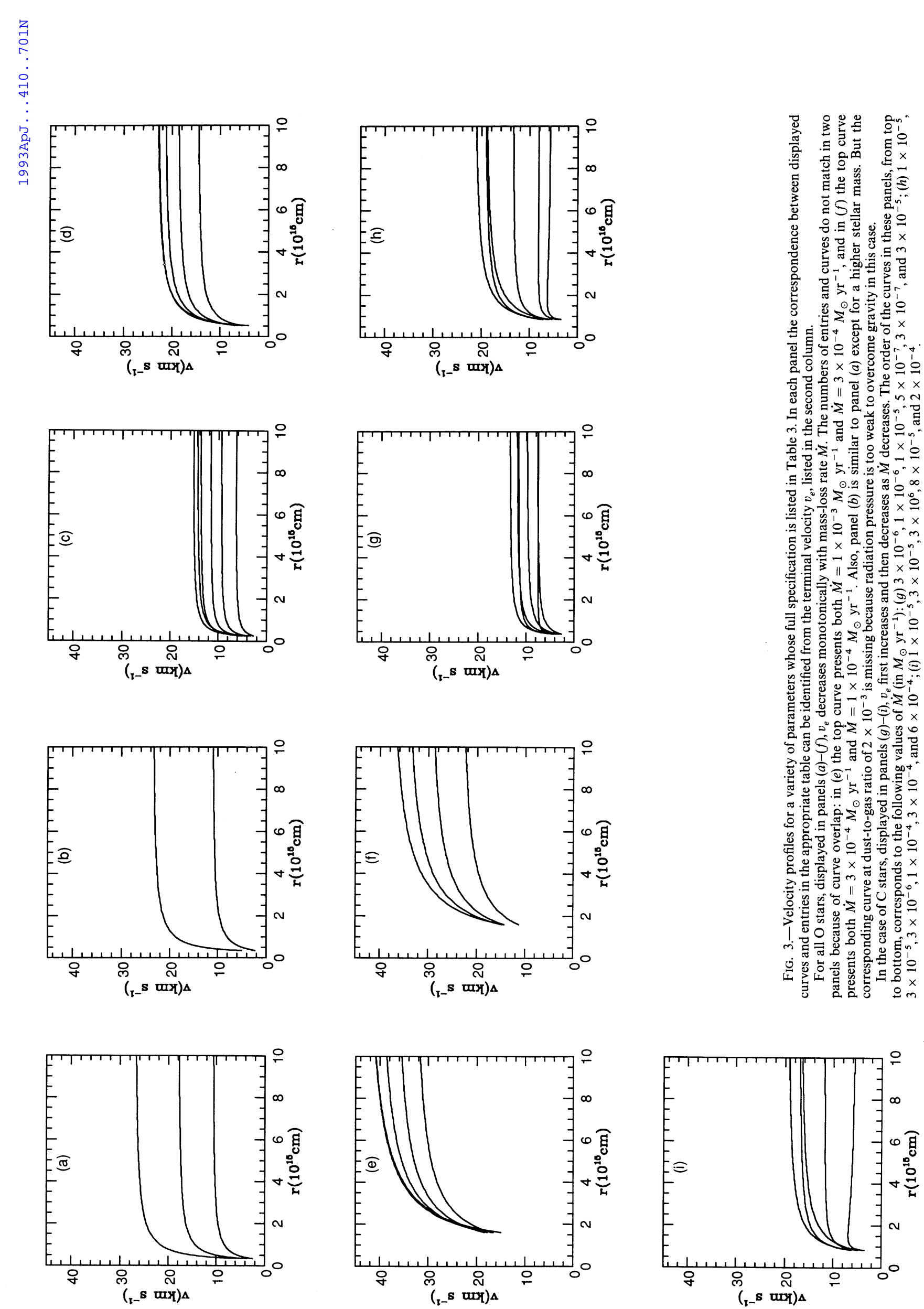
TABLE 3

Terminal Outflow Velocity, Optical Depths at 1 and 10 Microns, and Flux Mean Optical Depth for Model Stars Used for Figure 3

A. PANel $(a)$ :

O-star, $M=3 M_{\odot}, \dot{M}=3 \times 10^{-5} M_{\odot} \mathrm{yr}^{-1}$, $L=5 \times 10^{4} L_{\odot}, T_{e}=2,500 \mathrm{~K}$
F. Panel $(f)$ :

O-star, $M=30 M_{\odot}, L=4 \times 10^{5} L_{\odot}$, $T_{e}=2,500 \mathrm{~K}$, Dust-to-Gas Ratio $=0.005$

\begin{tabular}{|c|c|c|c|c|c|c|c|c|c|}
\hline Dust-to-Gas & $\left(\mathrm{km} \mathrm{s}^{v_{e}}\right)$ & $\tau(1 \mu \mathrm{m})$ & $\tau(10 \mu \mathrm{m})$ & $\tau_{F}$ & $\begin{array}{c}\dot{M} \\
\left(M_{\odot} \mathrm{yr}^{-1}\right)\end{array}$ & $\left(\mathrm{km} \mathrm{s}^{v_{e}}\right)$ & $\tau(1 \mu \mathrm{m})$ & $\tau(10 \mu \mathrm{m})$ & $\tau_{F}$ \\
\hline $0.005 \ldots \ldots \ldots \ldots \ldots \ldots$ & 27. & 1.3 & 3.9 & 1.1 & \multirow{4}{*}{$\begin{array}{l}3 \times 10^{-4} \ldots \ldots \ldots \ldots \ldots \\
1 \times 10^{-4} \ldots \ldots \ldots \ldots \ldots \ldots \\
3 \times 10^{-5} \ldots \ldots \ldots \ldots \ldots \ldots \\
1 \times 10^{-5} \ldots \ldots \ldots \ldots \ldots\end{array}$} & \multirow{4}{*}{$\begin{array}{l}39 . \\
35 . \\
30 . \\
24 .\end{array}$} & \multirow{4}{*}{$\begin{array}{l}2.2 \\
0.76 \\
0.24 \\
0.08\end{array}$} & 6.4 & \multirow{4}{*}{$\begin{array}{l}1.8 \\
0.61 \\
0.18 \\
0.059\end{array}$} \\
\hline $0.003 \ldots \ldots \ldots \ldots \ldots \ldots$ & 18. & 1.2 & 3.4 & 0.9 & & & & 2.2 & \\
\hline $0.002 \ldots \ldots \ldots \ldots \ldots \ldots$ & 11. & 1.2 & 3.5 & 0.97 & & & & 0.69 & \\
\hline & B. PAN & & & & & & & 0.24 & \\
\hline
\end{tabular}

O-star, $M=5 M_{\odot}, \dot{M}=3 \times 10^{-5} M_{\odot} \mathrm{yr}^{-1}$, $L=5 \times 10^{4} L_{\odot}, T_{e}=2.500 \mathrm{~K}$

\begin{tabular}{|c|c|c|c|c|}
\hline Dust-to-Gas & $\begin{array}{c}v_{e} \\
\left(\mathrm{~km} \mathrm{~s}^{-1}\right)\end{array}$ & $\tau(1 \mu \mathrm{m})$ & $\tau(10 \mu \mathrm{m})$ & $\tau_{F}$ \\
\hline $0.005 \ldots \ldots \ldots \ldots \ldots \ldots$ & 24. & 1.5 & 4.4 & 1.2 \\
\hline $0.003 \ldots \ldots \ldots \ldots \ldots \ldots$ & 11. & 2.0 & 5.7 & 1.6 \\
\hline \multicolumn{5}{|c|}{$\begin{array}{c}\text { C. PANEL }(c): \\
\text { O-star, } M=1 M_{\odot}, L=1 \times 10^{4} L_{\odot} \\
T_{e}=3,000 \mathrm{~K} \text {, Dust-to-Gas Ratio }=0.005\end{array}$} \\
\hline $\begin{array}{c}\dot{M} \\
\left(M_{\odot} \mathrm{yr}^{-1}\right)\end{array}$ & $\begin{array}{c}v_{e} \\
\left(\mathrm{~km} \mathrm{~s}^{-1}\right)\end{array}$ & $\tau(1 \mu \mathrm{m})$ & $\tau(10 \mu \mathrm{m})$ & $\tau_{F}$ \\
\hline $3 \times 10^{-5}$. & 15.3 & 3.2 & 9.3 & 3.2 \\
\hline $1 \times 10^{-5}$ & 14.5 & 1.0 & 2.9 & 0.99 \\
\hline $3 \times 10^{-6}$. & 13.8 & 0.33 & 0.94 & 0.31 \\
\hline $1 \times 10^{-6}$. & 11.7 & 0.11 & 0.32 & 0.11 \\
\hline $5 \times 10^{-7}$ & 9.5 & 0.056 & 0.16 & 0.054 \\
\hline $3 \times 10^{-7} \ldots \ldots \ldots \ldots \ldots$ & 6.4 & 0.038 & 0.11 & 0.037 \\
\hline
\end{tabular}

D. PANEl $(d)$ :

O-star, $M=4 M_{\odot}, L=5 \times 10^{4} L_{\odot}$,

$T_{e}=2,500 \mathrm{~K}$, Dust-to-Gas Ratio $=0.005$

\begin{tabular}{ccccc}
\hline \hline $\begin{array}{c}\dot{M} \\
\left(M_{\odot} \mathrm{yr}^{-1}\right)\end{array}$ & $\begin{array}{c}v_{e} \\
\left(\mathrm{~km} \mathrm{~s}^{-1}\right)\end{array}$ & $\tau(1 \mu \mathrm{m})$ & $\tau(10 \mu \mathrm{m})$ & $\tau_{\boldsymbol{F}}$ \\
\hline $3 \times 10^{-4} \ldots \ldots \ldots \ldots \ldots$. & 23. & 11. & 31. & 8.3 \\
$1 \times 10^{-4} \ldots \ldots \ldots \ldots \ldots$. & 23. & 3.6 & 10. & 3.2 \\
$3 \times 10^{-5} \ldots \ldots \ldots \ldots \ldots$. & 22. & 1.1 & 3.3 & 0.94 \\
$1 \times 10^{-5} \ldots \ldots \ldots \ldots \ldots$. & 19. & 0.39 & 1.1 & 0.31 \\
$3 \times 10^{-6} \ldots \ldots \ldots \ldots \ldots$ & 15. & 0.12 & 0.36 & 0.09 \\
\hline
\end{tabular}

E. PANel (e):

O-star, $M=10 M_{\odot}, L=4 \times 10^{5} L_{\odot}$

$T_{e}=2,500 \mathrm{~K}$, Dust-to-Gas Ratio $=0.005$

\begin{tabular}{ccccc}
\hline \hline $\begin{array}{c}\dot{M} \\
\left(M_{\odot} \mathrm{yr}^{-1}\right)\end{array}$ & $\begin{array}{c}v_{e} \\
\left(\mathrm{~km} \mathrm{~s}^{-1}\right)\end{array}$ & $\tau(1 \mu \mathrm{m})$ & $\tau(10 \mu \mathrm{m})$ & \multicolumn{1}{c}{$\tau_{F}$} \\
\hline $1 \times 10^{-3} \ldots \ldots \ldots \ldots \ldots$ & 43. & 6.6 & 19. & 5.2 \\
$3 \times 10^{-4} \ldots \ldots \ldots \ldots \ldots$. & 43. & 2.0 & 5.7 & 1.6 \\
$1 \times 10^{-4} \ldots \ldots \ldots \ldots \ldots$. & 41. & 0.67 & 1.9 & 0.52 \\
$3 \times 10^{-5} \ldots \ldots \ldots \ldots \ldots$. & 37. & 0.20 & 0.58 & 0.15 \\
$1 \times 10^{-5} \ldots \ldots \ldots \ldots \ldots$ & 33. & 0.063 & 0.18 & 0.045 \\
\hline
\end{tabular}

velocity observed in the $\mathrm{OH} 1612 \mathrm{MHz}$ maser line, VX Sagittarii is located on the $v_{e}-\dot{M}$ diagram of Figure $2 b$ where the sign $V$ is marked. This location corresponds to a supergiant with normal metallicity, a mass of about $40-50 M_{\odot}$, and a luminosity of $4 \times 10^{5} L_{\odot}$ (its assumed luminosity and the one we used in all the calculations for supergiants). If the mass-loss rate were increased to about $10^{-4} M_{\odot} \mathrm{yr}^{-1}$ (which is not unusual for such supergiants) with the other parameters unchanged, the outflow velocity would become about $30 \mathrm{~km}$ $\mathrm{s}^{-1}$ - the observed CO velocity. It is possible that the mass- loss rate was this high in the past, producing the high outflow velocity of the observed CO layers. As the mass-loss rate decreased to its current value, the outflow velocity decreased to the observed value of the $1612 \mathrm{MHz} \mathrm{OH}$ maser line. Furthermore, Figure $2 b$ shows that the current mass loss rate of VX Sagittarii is not much higher than the minimum limit. The outflow velocity in that part of the diagram is very sensitive to changes in the mass-loss rate. Therefore, a minor decrease in the mass-loss rate during the crossing time between the $\mathrm{H}_{2} \mathrm{O}$ and $\mathrm{OH}$ maser shells, about $200 \mathrm{yr}$, could cause the difference 

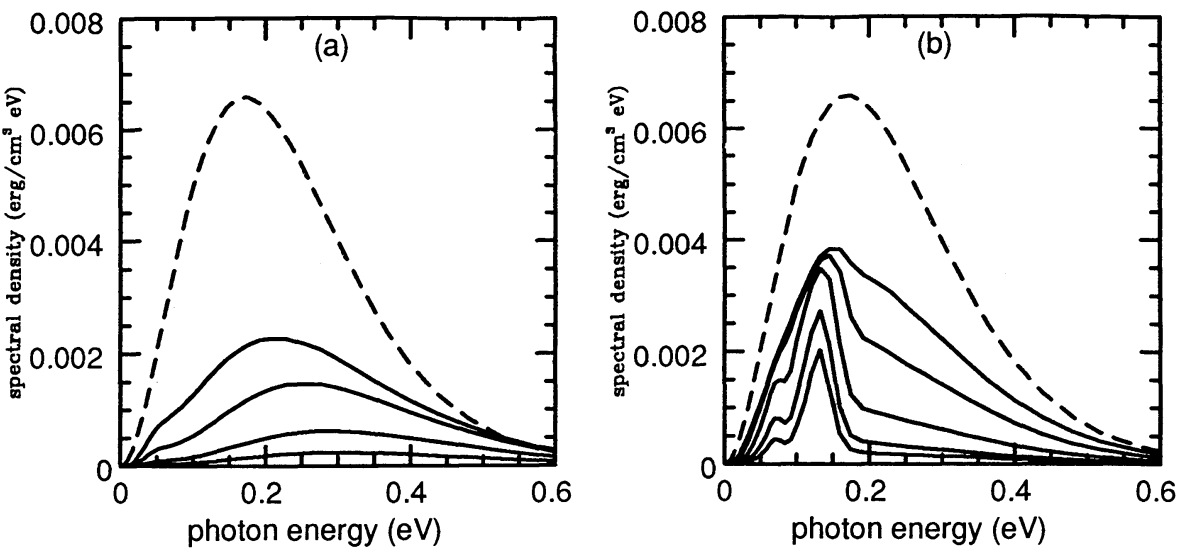

FIG. 4.-The spectral energy density in the central cavity inside the dust formation point. (a) Carbon stars with a mass of $4 M_{\odot}$, effective temperature of $2000 \mathrm{~K}$ and luminosity of $5 \times 10^{4} L_{\odot}$. The dust-to-gas ratio by mass is 0.0015 . The mass-loss rates are (solid lines, from bottom to top): $1 \times 10^{-5} M_{\odot} \mathrm{yr}^{-1}, 3 \times 10^{-5} M_{\odot}$ $\mathrm{yr}^{-1}, 1 \times 10^{-4} M_{\odot} \mathrm{yr}^{-1}, 3 \times 10^{-4} M_{\odot} \mathrm{yr}^{-1}$. The dashed line corresponds to a blackbody curve of $700 \mathrm{~K}$. (b) Oxygen stars with a mass of $4 M_{\odot}$, effective temperature of $2500 \mathrm{~K}$, and luminosity of $5 \times 10^{4} L_{\odot}$. The dust-to-gas ratio by mass is 0.005 . The mass-loss rates are (solid lines, from bottom to top): $3 \times 10^{-6} M_{\odot}$ $\mathrm{yr}^{-1}, 1 \times 10^{-5} M_{\odot} \mathrm{yr}^{-1}, 3 \times 10^{-5} M_{\odot} \mathrm{yr}^{-1}, 1 \times 10^{-4} M_{\odot} \mathrm{yr}^{-1}, 3 \times 10^{-4} M_{\odot} \mathrm{yr}^{-1}$. The dashed line corresponds to a blackbody curve of $700 \mathrm{~K}$.

between the observed outflow velocities of the two regions. Possibly, we are witnessing a stellar evolutionary effect involving decrease of the mass-loss rate.

\section{CONCLUSIONS}

Our results show that radiative transfer effects provide satisfactory explanations for dynamical properties of circumstellar envelopes. Dust drift through the gas has a major impact on the opacity and leads to a lower limit on the mass-loss rates that can be driven by radiation pressure on dust. This limit is of order $10^{-7} M_{\odot} \mathrm{yr}^{-1}$, close to the value observed for Mira variables. Lower mass-loss rates are characterized by lower outflow velocities and must be attributed to other processes.

Differences in dust optical properties lead to different outflow characteristics in carbon stars and oxygen stars, as the stellar radiation is reprocessed differently in the two classes. The difference is most notable in high mass-loss rates. In carbon stars there is an upper limit of several times $10^{-4} \mathrm{yr}^{-1}$ on possible mass-loss rates, while the corresponding limit in oxygen stars is presumably higher and of no observational interest.
Major sources of uncertainty are the grain size distribution and the character of scattering. Our results suggest that grains with sizes of $0.3-2 \mu \mathrm{m}$ cannot dominate the envelope behavior. The silicate optical properties, along with the dust drift, may explain some of the gradual acceleration observed in the outflow around red supergiants.

We thank B. Draine for providing us with updated tables of optical properties of dust grains, F. Shu for a revealing comment about the solution of the radiative transfer problem, and $\mathrm{S}$. Beck for providing us with results of observations. We also thank B. Paczyński, P. Bowers, H. Johnson, N. Anderson, P. Lockett, and C. Gammie for fruitful discussions. Nathan Netzer wishes to thank the Astronomy Department of Indiana University for lending him the use of the software needed to prepare this paper. We are particularly thankful to G. Knapp, for drawing our attention to rich observational material that we used for comparing the results of calculations with observations. This work was supported in part by NSF grant AST9016810 and by the center for Computational Sciences of the University of Kentucky.

\section{REFERENCES}

Adams, F. C., \& Shu, F. H. 1986, ApJ, 308, 836

Arquilla, R., Leahy, D. A., \& Kwok, S. 1986, MNRAS, 220, 125

Baud, B., Habing, H. J., Matthews, H. E., \& Winnberg, A. 1981, A\&A, 95, 156

Bowers, P. F. 1984, in Mass Loss from Red Giants, ed. M. Morris \& B.

Zuckerman (Dordrecht: Reidel), 189

Bowers, P. F., \& Johnston, K. J. 1990, ApJ, 354, 676

Chapman, J. M., \& Cohen, R. J. 1986, MNRAS, 220, 513 (CC)

Deguchi, S. 1980, ApJ, 236, 567

Draine, B. T. 1987, Princeton University Observatory preprint 213

Draine, B. T., \& Lee, H. M. 1984, ApJ, 285, 89

Draine, B. T., \& Salpeter, E. E. 1979, ApJ, 231, 77

Gail, H.-P., \& Sedlmayr, E. 1987, A\&A, 177, 186

Gilman, R.C. 1972, ApJ, 178, 473

Goldreich, P., \& Scoville, N. 1976, ApJ, 205, 144

Hagen, W., Stencel, R. E., \& Dickinson, D. F. 1983, ApJ, 274, 286

Jura, M. 1984, ApJ, 282, 200

Jura, M., Kahane, C., \& Omont, A. 1988, A\&A, 201, 80

Knapp, G. R. 1985, ApJ, 293, 273 1986, ApJ, 311, 731 1991, private communication

Knapp, G. R., \& Morris, M. 1985, ApJ, 292, 640

Knapp, G. R., Phillips T. G., Leighton, R. B., Lo, K. Y., Wannier, P. G. Wootten, H. A., \& Huggins, P. J. 1982, ApJ, 252, 616

Knapp, G. R., et al. 1989, ApJ, 336, 822

Kwok, S. 1975, ApJ, 198, 583

Leahy, D. A., Kwok, S., \& Arquilla, R. A. 1987, ApJ, 320, 825
Le Bertre, T. 1987, A\&A, 176, 107

Le Borgne, J. F., \& Mauron, N. 1989, A\&A, 210, 198

Leung, C. M. 1975, ApJ, 199, 340

Likkel, L., Forveille, T., Omont, A., \& Morris, M. 1988, A\&A, 198, L1

Likkel, L., Omont, A., Morris, M., \& Forveille, T. 1987, A\&A, 173, L11

Netzer, N. 1989, ApJ, 342, 1068

Olofsson, H. Eriksson, K., \& Gustafsson, B. 1987, A\&A, 183, L13

. 1988, A\&A, 196, L1

Papoular, E., \& Pegourie, B. 1983, A\&A, 128, 335

Rieu, N. Q., Epchtein, N., Bach, T., \& Cohen, M. 1987, A\&A, 180, 117

Rowan-Robinson, M. 1980, ApJS, 44, 403

Rowan- Robinson, M., \& Harris, S. 1982, MNRAS, 200, 197

1983, MNRAS, 202, 767

Salpeter, E. E. 1974, ApJ, 193, 585

Schwartzschild, M. 1958, Structure and Evolution of Stars (Princeton: Princeton Univ. Press)

Snow, T. P., Buss, R. H., Gilra, D. P., \& Swings, J.-P. 1987, ApJ, 321, 921

van de Hulst, H. C. 1957, Light Scattering by Small Particles (New York: Dover)

Wannier, P. G., \& Sahai, R. 1986, ApJ, 311, 335

Welch, W. J. 1989, in The Physics and Chemistry of Interstellar Molecular Clouds, ed. G. Winnewisser \& J. T. Armstrong (Berlin: Springer-Verlag), 5

Zuckerman, B., \& Dyck, H. M. 1986a, ApJ, 304, 394

1986b, ApJ, 311, 345

. 1989, A\&A, 209, 119

Zuckerman, B., Dyck, H. M., \& Claussen, M. J. 1986, ApJ, 304, 401 